\title{
Palladium-catalyzed cross-coupling between vinyl halides and tert-butyl carbazate: First general synthesis of the unusual $N$-Boc- $N$-alquenylhydrazines
}

\author{
José Barluenga*, Patricia Moriel, Fernando Aznar, Carlos Valdés* \\ Instituto Universitario de Química Organometálica "Enrique Moles", Universidad de Oviedo, Julián \\ Clavería 8, 33006 Oviedo, Spain
}

SUPPORTING INFORMATION

General considerations: All reactions were carried out under nitrogen atmosphere in a RR98030 12 place Carousel Reaction Station ${ }^{\mathrm{TM}}$ from Radleys Discovery Technologies, equipped with gas-tight threaded caps with a valve, cooling reflux head system, and digital temperature controller. DMF, dichloromethane and hexanes were dehydrated using the procedures described in D. Perrin Purification of Laboratory Chemicals, Pergamon Press Ltd. 1980, $2^{\text {nd }}$ Ed. $\mathrm{Pd}_{2}(\mathrm{dba})_{3}$ was purchased from Strem Chemical co. and used without further purification. All ligands used are commercially available from Strem Chemical co. or Aldrich Chemical co. and were used without further purification. $\mathrm{Cs}_{2} \mathrm{CO}_{3}$ was purchased from Aldrich Chemical co., stored in a flask purged with nitrogen and weighted in the air. tert-Butyl carbazate is commercially available from Acros Organics and was used without further purification. $\beta$-bromostyrene is commercially available from Aldrich Chemical co. and was used after being distilled. GC analysis were performed with a GC Agilent Technologies 6890N instrument. NMR spectra were recorded at 400,300 or $200 \mathrm{MHz}$ for ${ }^{1} \mathrm{H}$ and 100,75 or $50 \mathrm{MHz}$ for ${ }^{13} \mathrm{C}$, with tetramethylsilane as internal standard for ${ }^{1} \mathrm{H}$ and the residual solvent signals as standard for ${ }^{13} \mathrm{C}$. Chemical shifts are given in ppm. Mass spectra were obtained by EI $(70 \mathrm{eV})$ or FAB-LR. 
Preparation of bromoalkenes $2 \mathrm{~b}, 2 \mathrm{c}$ and $2 \mathrm{i}$ :

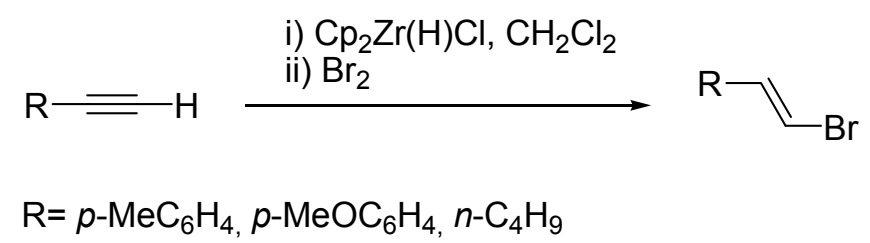

To the stirred suspension of $24 \mathrm{mmol}$ of $\mathrm{Cp}_{2} \mathrm{Zr}(\mathrm{H}) \mathrm{Cl}$ in $30 \mathrm{~mL}$ THF under $\mathrm{N}_{2}$ atmosphere, and in a flask protected from the sun light, were added dropwise $20 \mathrm{mmol}$ of the corresponding alkyne dissolved in $10 \mathrm{~mL}$ of THF. The mixture was stirred at room temperature for 3 hours. The solution was cooled to $0^{\circ} \mathrm{C}$ and then $20 \mathrm{mmol}$ of bromine were added dropwise. After stirring for $10 \mathrm{~min}$, the reaction was quenched with $20 \mathrm{~mL}$ of saturated aqueous $\mathrm{Na}_{2} \mathrm{~S}_{2} \mathrm{O}_{3}$ solution. The organics were extracted with diethyl ether $(2 \times 30 \mathrm{~mL})$, the organic layers were combined, washed with brine and dried over $\mathrm{Na}_{2} \mathrm{SO}_{4}$. Removal of the solvent under reduced pressure afforded the bromoalkenes as nearly pure materials, which were further purified by filtration through a short chromatographic column.

Preparation of bromoalkenes 2d, 2e, 2 f and 2g: These bromoalkenes were prepared following the procedure described in S. Abbas, C. J. Hayes, S. Worden, Tetrahedron Lett. 2000, 41, 3215-3219.

Preparation of bromoalkene 2h: This bromoalkene was prepared following the procedure described in E.-i Negishi, M. Hata, C. Xu, Org. Lett. 2000, 2, 3687-3689.

Preparation of chlorodienes $\mathbf{2} \mathbf{j}$ and $\mathbf{2 k}$ : These chlorodienes were prepared according to the procedure described in J. Barluenga, P. Moriel, F. Aznar, C. Valdés, Adv. Synth. Cat. 2006, 348, 347-353.

General procedure for the cross-coupling of tert-butyl carbazate 1 with alkenyl halides 2a-j. A carousel reaction tube under nitrogen atmosphere was charged with JOHNPHOS (0.08 mmol, $8 \mathrm{~mol} \%)$, tris(dibenzylideneacetone)dipalladium (0) (0.02 mmol, $4 \mathrm{~mol} \% \mathrm{Pd})$, cesium carbonate $(1.4 \mathrm{mmol})$, tert-butyl carbazate $1(1.2 \mathrm{mmol})$ and DMF ( $2 \mathrm{~mL})$. After stirring the mixture for a minute, the haloalkene $\mathbf{2 a - j}(1 \mathrm{mmol})$ was added. The system was heated at $110^{\circ} \mathrm{C}\left(90^{\circ} \mathrm{C}\right.$ for $\left.\mathbf{2 d}\right)$ with stirring until the starting halide had been completely consumed as judged by GC analysis. The mixture was 
allowed to cool to room temperature, taken up in dry dichloromethane $(15 \mathrm{~mL})$, and filtered through Celite. The solvents were removed under reduced pressure. The residue was purified by flash chromatography.

Using this procedure were prepared the following compounds:

$N$-tert-butoxycarbonyl- $N$ - $[(E)-2$-phenylethenyl]hydrazine (3a):<smiles>CC(=O)OC(=O)N(N)/C=C/c1ccccc1</smiles>

HRMS (EI): calcd. for $\mathrm{C}_{13} \mathrm{H}_{18} \mathrm{~N}_{2} \mathrm{O}_{2}$ : 234.1368; found: 234.1369; ${ }^{1} \mathrm{H}$ NMR ( $\mathrm{CDCl}_{3}, 200$ $\mathrm{MHz}): \delta=1.58(\mathrm{~s}, 9 \mathrm{H}), 4.16$ (br. s, $2 \mathrm{H}), 6.39\left(\mathrm{~d},{ }^{3} J_{\text {trans }}=14.1 \mathrm{~Hz}, 1 \mathrm{H}\right), 7.12-7.21(\mathrm{~m}$, 1H), 7.26-7.33 (m, 5H); ${ }^{13} \mathrm{C} \mathrm{NMR}\left(\mathrm{CDCl}_{3}, 50 \mathrm{MHz}\right): \delta=28.2\left(\mathrm{CH}_{3}\right), 82.4(\mathrm{C}), 109.2$ $(\mathrm{CH}), 125.3(\mathrm{CH}), 125.72(\mathrm{CH}), 127.2(\mathrm{CH}), 128.5(\mathrm{CH}), 137.0(\mathrm{C}), 153.2(\mathrm{C})$.

$N$-tert-butoxycarbonyl- $N$-[(E)-2-(p-tolyl)ethenyl]hydrazine (3b):<smiles>Cc1ccc(/C=C/N(N)C(=O)O[Na])cc1</smiles>

HRMS (EI): calcd. for $\mathrm{C}_{14} \mathrm{H}_{20} \mathrm{~N}_{2} \mathrm{O}_{2}$ : 248.1519; found: 248.1526; ${ }^{1} \mathrm{H}$ NMR $\left(\mathrm{CDCl}_{3}, 300\right.$ MHz): $\delta=1.59(\mathrm{~s}, 9 \mathrm{H}), 2.37(\mathrm{~s}, 3 \mathrm{H}), 3.80$ (br. s, $2 \mathrm{H}), 6.35\left(\mathrm{~d},{ }^{3} J_{\text {trans }}=14.1 \mathrm{~Hz}, 1 \mathrm{H}\right)$, 7.00-7.26 (m, 5H); ${ }^{13} \mathrm{C} \mathrm{NMR}\left(\mathrm{CDCl}_{3}, 75 \mathrm{MHz}\right): 21.0\left(\mathrm{CH}_{3}\right), 28.2\left(\mathrm{CH}_{3}\right), 82.3(\mathrm{C})$, $109.2(\mathrm{CH}), 125.2(\mathrm{CH}), 126.5(\mathrm{CH}), 129.0(\mathrm{CH}), 134.1(\mathrm{C}), 135.4(\mathrm{C}), 153.2(\mathrm{C})$.

$N$-tert-butoxycarbonyl- $N$ - $[(E)-2-(p$-methoxyphenyl)ethenyl]hydrazine (3c):<smiles>COc1ccc(/C=C/N(N)C(=O)OCc2ccccc2)cc1</smiles>

HRMS (EI): calcd. for $\mathrm{C}_{14} \mathrm{H}_{20} \mathrm{~N}_{2} \mathrm{O}_{3}$ : 264.1468; found: 264.1463; ${ }^{1} \mathrm{H}$ NMR $\left(\mathrm{CDCl}_{3}, 300\right.$ MHz): $\delta=1.59$ (s, 9H), 3.83 (br. s, $5 \mathrm{H}), 6.37\left(\mathrm{~d},{ }^{3} J_{\text {trans }}=14.0 \mathrm{~Hz}, 1 \mathrm{H}\right), 6.88\left(\mathrm{~d},{ }^{3} J=8.7\right.$ $\mathrm{Hz}, 2 \mathrm{H}), 7.27-7.31(\mathrm{~m}, 3 \mathrm{H}) ;{ }^{13} \mathrm{C} \mathrm{NMR}\left(\mathrm{CDCl}_{3}, 75 \mathrm{MHz}\right): \delta=28.2\left(\mathrm{CH}_{3}\right), 55.2\left(\mathrm{CH}_{3}\right)$, $82.3(\mathrm{C}), 109.0(\mathrm{CH}), 114.0(\mathrm{CH}), 125.7(\mathrm{CH}), 126.4(\mathrm{CH}), 129.7(\mathrm{C}), 153.3(\mathrm{C}), 157.9$ (C). 

(3d):<smiles>COC(=O)c1ccc(/C=C/N(N)C(=O)OCc2ccccc2)cc1</smiles>

HRMS (EI): calcd. for $\mathrm{C}_{15} \mathrm{H}_{20} \mathrm{~N}_{2} \mathrm{O}_{4}$ : 292.1418; found: 292.1414; ${ }^{1} \mathrm{H}$ NMR $\left(\mathrm{CDCl}_{3}, 300\right.$ $\mathrm{MHz}): \delta=1.55(\mathrm{~s}, 9 \mathrm{H}), 3.88$ (br. s, 5H), $6.38\left(\mathrm{~d},{ }^{3} J_{\text {trans }}=14.2 \mathrm{~Hz}, 1 \mathrm{H}\right), 7.25-7.34(\mathrm{~m}$, $3 \mathrm{H}), 7.92\left(\mathrm{~d},{ }^{3} \mathrm{~J}=8.2 \mathrm{~Hz}, 2 \mathrm{H}\right) ;{ }^{13} \mathrm{C} \mathrm{NMR}\left(\mathrm{CDCl}_{3}, 75 \mathrm{MHz}\right): \delta=28.1\left(\mathrm{CH}_{3}\right), 51.8\left(\mathrm{CH}_{3}\right)$, $82.9(\mathrm{C}), 108.4(\mathrm{CH}), 124.9(\mathrm{CH}), 126.8(\mathrm{C}), 126.9(\mathrm{CH}), 129.9(\mathrm{CH}), 142.0(\mathrm{C}), 153.0$ (C), $166.9(\mathrm{C})$.

\section{$N$-tert-butoxycarbonyl- $N-[(E)-2-(p$-chlorophenyl)ethenyl]hydrazine (3e):}<smiles>NN(C=Cc1ccc(Cl)cc1)C(=O)O</smiles>

HRMS (FAB) $(\mathrm{M}+1)^{+}$: calcd. for $\mathrm{C}_{13} \mathrm{H}_{18} \mathrm{ClN}_{2} \mathrm{O}_{2}$ : 269.1057; found: 269.1064; ${ }^{1} \mathrm{H} \mathrm{NMR}$ $\left(\mathrm{CDCl}_{3}, 400 \mathrm{MHz}\right): \delta=1.56(\mathrm{~s}, 9 \mathrm{H}), 3.98($ br. s, $2 \mathrm{H}), 6.33\left(\mathrm{~d},{ }^{3} J_{\text {trans }}=14.1 \mathrm{~Hz}, 1 \mathrm{H}\right)$, 7.24-7.32 (m, 5H); ${ }^{13} \mathrm{C} \mathrm{NMR}\left(\mathrm{CDCl}_{3}, 100 \mathrm{MHz}\right): \delta=28.3\left(\mathrm{CH}_{3}\right), 82.8(\mathrm{C}), 108.2(\mathrm{CH})$, 126.6 (CH), $127.8(\mathrm{C}), 128.7(\mathrm{CH}), 131.2(\mathrm{C}), 135.8(\mathrm{CH}), 153.3(\mathrm{C})$.

$N$-tert-butoxycarbonyl- $N$ - $[(E)-2-(o$-bromophenyl)ethenyl]hydrazine (3f):<smiles>CC(=O)OC(=O)N(N)/C=C/c1ccccc1Br</smiles>

HRMS (EI): calcd. for $\mathrm{C}_{13} \mathrm{H}_{17} \mathrm{BrN}_{2} \mathrm{O}_{2}$ : 312.0468; found: 312.0456; ${ }^{1} \mathrm{H}$ NMR $\left(\mathrm{CDCl}_{3}\right.$, $300 \mathrm{MHz}): \delta=1.55(\mathrm{~s}, 9 \mathrm{H}), 4.16$ (br. s, $2 \mathrm{H}), 6.66\left(\mathrm{~d},{ }^{3} J_{\text {trans }}=14.1 \mathrm{~Hz}, 1 \mathrm{H}\right), 7.01\left(\mathrm{t},{ }^{3} \mathrm{~J}=\right.$ $7.6 \mathrm{~Hz}, 1 \mathrm{H}), 7.23\left(\mathrm{t},{ }^{3} J=7.6 \mathrm{~Hz}, 1 \mathrm{H}\right), 7.40-7.43(\mathrm{~m}, 2 \mathrm{H}), 7.53\left(\mathrm{~d},{ }^{3} J=8.0 \mathrm{~Hz}, 1 \mathrm{H}\right) ;{ }^{13} \mathrm{C}$ NMR $\left(\mathrm{CDCl}_{3}, 75 \mathrm{MHz}\right): \delta=28.2\left(\mathrm{CH}_{3}\right), 82.7(\mathrm{C}), 108.4(\mathrm{CH}), 123.2(\mathrm{C}), 125.7(\mathrm{CH})$, $127.1(\mathrm{CH}), 127.3(\mathrm{CH}), 129.4(\mathrm{CH}), 132.9(\mathrm{CH}), 137.0(\mathrm{C}), 153.1(\mathrm{C})$. 
$N$-tert-butoxycarbonyl- $N$-[(1E,3E)-4-phenyl-1,3-butadienyl]hydrazine (3g):<smiles>CC(=O)OC(=O)N(N)/C=C/C=C/c1ccccc1</smiles>

HRMS (EI): calcd. for $\mathrm{C}_{15} \mathrm{H}_{20} \mathrm{~N}_{2} \mathrm{O}_{2}$ : 260.1519; found: 260.1512; ${ }^{1} \mathrm{H}$ NMR $\left(\mathrm{CDCl}_{3}, 300\right.$ MHz): $\delta=1.54$ (s, 9H), 3.99 (br. s, $2 \mathrm{H}), 6.25$ (dd, $\left.{ }^{3} J_{\text {trans }}=13.4 \mathrm{~Hz},{ }^{3} J=10.8 \mathrm{~Hz}, 1 \mathrm{H}\right)$, $6.46\left(\mathrm{~d},{ }^{3} J_{\text {trans }}=15.7 \mathrm{~Hz}, 1 \mathrm{H}\right), 6.81\left(\mathrm{dd},{ }^{3} J_{\text {trans }}=15.7 \mathrm{~Hz},{ }^{3} \mathrm{~J}=10.8 \mathrm{~Hz}, 1 \mathrm{H}\right), 7.18\left(\mathrm{~d},{ }^{3} \mathrm{~J}=\right.$ 7.1 Hz, 1H), 7.27-7.39 (m, 5H); ${ }^{13} \mathrm{C} \mathrm{NMR}\left(\mathrm{CDCl}_{3}, 75 \mathrm{MHz}\right): \delta=30.7\left(\mathrm{CH}_{3}\right), 85.1(\mathrm{C})$, $113.0(\mathrm{CH}), 128.2(\mathrm{CH}), 129.0(\mathrm{CH}), 129.8(\mathrm{CH}), 131.0(2 \times \mathrm{CH}), 133.1(\mathrm{CH}), 140.4(\mathrm{C})$, $155.4(\mathrm{C})$.

$N$-tert-butoxycarbonyl- $N-[(E)-1-($ dodec-1-en-3-ynyl) $]$ hydrazine (3h):

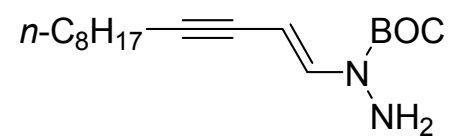

HRMS (EI): calcd. for $\mathrm{C}_{17} \mathrm{H}_{30} \mathrm{~N}_{2} \mathrm{O}_{2}$ : 294.2302; found: 294.2298; ${ }^{1} \mathrm{H}$ NMR $\left(\mathrm{CDCl}_{3}, 400\right.$ $\mathrm{MHz}): \delta=0.88\left(\mathrm{t},{ }^{3} J=6.8 \mathrm{~Hz}, 3 \mathrm{H}\right), 1.25-1.35(\mathrm{~m}, 9 \mathrm{H}), 1.36-1.40(\mathrm{~m}, 1 \mathrm{H}), 1.50(\mathrm{~s}, 11 \mathrm{H})$, $2.30\left(\mathrm{t},{ }^{3} J=7.0 \mathrm{~Hz}, 2 \mathrm{H}\right), 4.00$ (br. s, 2H), 5.42 (dt, $\left.{ }^{3} J=13.7 \mathrm{~Hz},{ }^{5} J=2.3 \mathrm{~Hz}, 1 \mathrm{H}\right), 7.19$ $\left(\mathrm{d},{ }^{3} J=13.7 \mathrm{~Hz}, 1 \mathrm{H}\right) ;{ }^{13} \mathrm{C} \mathrm{NMR}\left(\mathrm{CDCl}_{3}, 100 \mathrm{MHz}\right): \delta=14.0\left(\mathrm{CH}_{3}\right), 19.5\left(\mathrm{CH}_{2}\right), 22.6$ $\left(\mathrm{CH}_{2}\right), 28.1\left(\mathrm{CH}_{3}\right), 28.8\left(\mathrm{CH}_{2}\right), 28.9\left(\mathrm{CH}_{2}\right), 29.0\left(\mathrm{CH}_{2}\right), 29.1\left(\mathrm{CH}_{2}\right), 31.7\left(\mathrm{CH}_{2}\right), 77.7$ (C), $82.7(\mathrm{C}), 88.7(\mathrm{CH}), 89.2(\mathrm{C}), 136.4(\mathrm{CH}), 152.5(\mathrm{C})$.

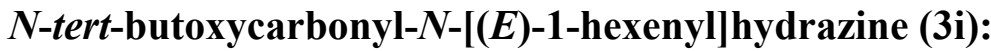

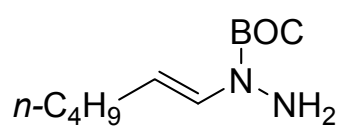

HRMS (EI): calcd. for $\mathrm{C}_{11} \mathrm{H}_{22} \mathrm{~N}_{2} \mathrm{O}_{2}$ : 214.1681; found: 214.1685; ${ }^{1} \mathrm{H}$ NMR $\left(\mathrm{CDCl}_{3}, 300\right.$ MHz): $\delta=0.85-0.90(\mathrm{~m}, 3 \mathrm{H}), 1.32-1.33(\mathrm{~m}, 4 \mathrm{H}), 1,48$ (s, 9H), 2.00-2.03 (m, 2H), 3.98 (br. s, $2 \mathrm{H}), 5.28\left(\mathrm{dt},{ }^{3} J_{\text {trans }}=14.1 \mathrm{~Hz},{ }^{3} J=7.3 \mathrm{~Hz}, 1 \mathrm{H}\right), 6.65-6.70(\mathrm{~m}, 1 \mathrm{H}) ;{ }^{13} \mathrm{C} \mathrm{NMR}$ $\left(\mathrm{CDCl}_{3}, 75 \mathrm{MHz}\right): \delta=13.8\left(\mathrm{CH}_{3}\right), 22.1\left(\mathrm{CH}_{2}\right), 28.2\left(\mathrm{CH}_{3}\right), 29.4\left(\mathrm{CH}_{2}\right), 32.5\left(\mathrm{CH}_{2}\right), 81.6$ (C), $109.4(\mathrm{CH}), 126.5(\mathrm{CH}), 153.2(\mathrm{C})$. 
$N$-tert-butoxycarbonyl- $N$-[(1E,3E)-deca-1,3-dienyl]hydrazine (3j):<smiles>CCCCCCC=CC=CC=CN(N)C(=O)OCc1ccccc1</smiles>

HRMS (EI): calcd. for $\mathrm{C}_{15} \mathrm{H}_{28} \mathrm{~N}_{2} \mathrm{O}_{2}$ : 268.2145; found: 268.2144; ${ }^{1} \mathrm{H}$ NMR $\left(\mathrm{CDCl}_{3}, 400\right.$ $\mathrm{MHz}): \delta=0.88\left(\mathrm{t},{ }^{3} J=6.7 \mathrm{~Hz}, 3 \mathrm{H}\right), 1.26-1.38(\mathrm{~m}, 8 \mathrm{H}), 1.50(\mathrm{~s}, 9 \mathrm{H}), 2.06\left(\mathrm{c},{ }^{3} J=7.0\right.$ $\mathrm{Hz}, 2 \mathrm{H}), 3.97$ (br. s, 2H), 5.57 (dt, , $\left.{ }^{3} J_{\text {trans }}=13.8 \mathrm{~Hz},{ }^{3} J=7.0 \mathrm{~Hz}, 1 \mathrm{H}\right), 5.97-6.06(\mathrm{~m}$, 2H), 6.78-7.07 (m, 1H); ${ }^{13} \mathrm{C}$ NMR $\left(\mathrm{CDCl}_{3}, 100 \mathrm{MHz}\right): 14.0\left(\mathrm{CH}_{3}\right), 22.5\left(\mathrm{CH}_{2}\right), 28.1$ $\left(\mathrm{CH}_{3}\right), 28.7\left(\mathrm{CH}_{2}\right), 29.4\left(\mathrm{CH}_{2}\right), 31.6\left(\mathrm{CH}_{2}\right), 32.7\left(\mathrm{CH}_{2}\right), 82.0(\mathrm{C}), 110.4(\mathrm{CH}), 127.6$ $(\mathrm{CH}), 127.9(\mathrm{CH}), 131.2(\mathrm{CH}), 153.2(\mathrm{C})$.

\section{$N$-tert-butoxycarbonyl- $N$-[(1E,3E)-4-(4-chlorophenyl)buta-1,3-dienyl]hydrazine} (3k):<smiles>CC(=O)N(N)/C=C/C=C/c1ccc(Cl)cc1</smiles>

HRMS (EI): calcd. for $\mathrm{C}_{15} \mathrm{H}_{19} \mathrm{ClN}_{2} \mathrm{O}_{2}$ : 294.1129; found: 294.1125; ${ }^{1} \mathrm{H}$ NMR $\left(\mathrm{CDCl}_{3}\right.$, $400 \mathrm{MHz}): \delta=1.52$ (s, 9H), 4.09 (br. s, $2 \mathrm{H}), 6.21$ (dd, ${ }^{3} J_{\text {trans }}=13.2 \mathrm{~Hz},{ }^{3} J_{\text {cis }}=11.0 \mathrm{~Hz}$, $1 \mathrm{H}), 6.36\left(\mathrm{~d},{ }^{3} J_{\text {trans }}=15.5 \mathrm{~Hz}, 1 \mathrm{H}\right), 6.74\left(\mathrm{dd},{ }^{3} J_{\text {trans }}=15.5 \mathrm{~Hz},{ }^{3} J_{\text {cis }}=11.0 \mathrm{~Hz}, 1 \mathrm{H}\right), 7.21-$ $7.28(\mathrm{~m}, 5 \mathrm{H}) ;{ }^{13} \mathrm{C} \mathrm{NMR}\left(\mathrm{CDCl}_{3}, 100 \mathrm{MHz}\right): 28.1\left(\mathrm{CH}_{3}\right), 82.6(\mathrm{C}), 110.1(\mathrm{CH}), 126.8$ $(\mathrm{CH}), 126.9(\mathrm{CH}), 128.0(\mathrm{CH}) ; 128.5(\mathrm{CH}), 131.1(\mathrm{CH}), 131.8(\mathrm{C}), 136.5(\mathrm{C}), 153.0$ (C).

General procedure for the homocoupling of alkenyl bromides $2 \mathrm{a}, 2 \mathrm{~b}, 2 \mathrm{~d}$ and $2 \mathrm{~g}$. A carousel reaction tube under nitrogen atmosphere was charged with Verkade's proazaphosphatrane $9(0.08 \mathrm{mmol}, 8 \mathrm{~mol} \%)$, tris(dibenzylideneacetone)dipalladium (0) (0.02 mmol, $4 \mathrm{~mol} \% \mathrm{Pd})$, cesium carbonate $(0.7 \mathrm{mmol})$, and DMF (1 mL). After stirring the mixture for a minute, the bromoalkene $\mathbf{2 a}, \mathbf{2 b}, \mathbf{2 d}$ and $\mathbf{2 g}(1 \mathrm{mmol})$ was added. The system was heated at $110^{\circ} \mathrm{C}\left(90^{\circ} \mathrm{C}\right.$ for $\left.\mathbf{2 d}\right)$ with stirring until the starting halide had been completely consumed as judged by GC analysis. The mixture was allowed to cool to room temperature, taken up in dry hexanes $(15 \mathrm{~mL})$, and filtered through Celite. The solvents were evaporated under reduced pressure. The residue was purificated by flash chromatography.

Using this procedure were prepared the following compounds: 
(1E,3E)-1,4-diphenylbuta-1,3-diene (4a):

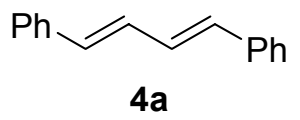

HRMS (EI): calcd. for $\mathrm{C}_{16} \mathrm{H}_{14}$ : 206.1096; found: 206.1097; ${ }^{1} \mathrm{H}$ NMR $\left(\mathrm{CDCl}_{3}, 300\right.$ $\mathrm{MHz}): \delta=6.70(\mathrm{~m}, 2 \mathrm{H}), 6.98(\mathrm{~m}, 2 \mathrm{H}), 7.23(\mathrm{~m}, 2 \mathrm{H}), 7.29(\mathrm{~m}, 4 \mathrm{H}), 7.37(\mathrm{~m}, 4 \mathrm{H}) ;{ }^{13} \mathrm{C}$ NMR $\left(\mathrm{CDCl}_{3}, 75 \mathrm{MHz}\right): \delta=126.3(\mathrm{CH}), 127.5(\mathrm{CH}), 128.6(\mathrm{CH}), 129.2(\mathrm{CH}), 132.7$ $(\mathrm{CH}), 137.2(\mathrm{C})$.

$(1 E, 3 E)-1,4-d i-p-t o l y l b u t a-1,3-d i e n e(4 b):$

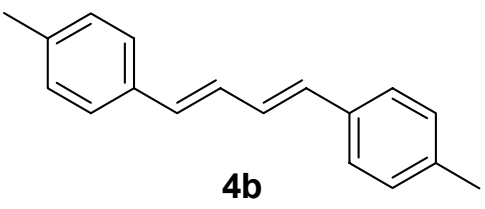

HRMS (EI): calcd. for $\mathrm{C}_{18} \mathrm{H}_{18}$ : 234.1409; found: 234.1920; ${ }^{1} \mathrm{H}$ NMR $\left(\mathrm{CDCl}_{3}, 400\right.$ $\mathrm{MHz}): \delta=2.37(\mathrm{~s}, 6 \mathrm{H}), 6.66-6.73(\mathrm{~m}, 2 \mathrm{H}), 6.91-6.95(\mathrm{~m}, 2 \mathrm{H}), 7.16\left(\mathrm{~d},{ }^{3} J=8.1 \mathrm{~Hz}\right.$, 4H), $7.36\left(\mathrm{~d},{ }^{3} J=8.1 \mathrm{~Hz}, 4 \mathrm{H}\right) ;{ }^{13} \mathrm{C} \mathrm{NMR}\left(\mathrm{CDCl}_{3}, 100 \mathrm{MHz}\right): \delta=21.2\left(\mathrm{CH}_{3}\right), 126.2$ $(\mathrm{CH}), 128.4(\mathrm{CH}), 129.3(\mathrm{CH}), 132.2(\mathrm{CH}), 134.6(\mathrm{C}), 137.3(\mathrm{C})$.

(1E,3E)-1,4-di(p-methoxycarbonylphenyl)buta-1,3-diene (4d):

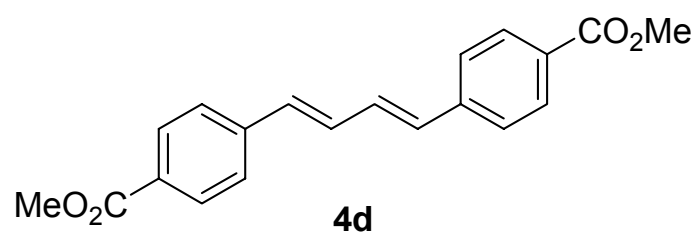

HRMS (EI): calcd. for $\mathrm{C}_{20} \mathrm{H}_{18} \mathrm{O}_{4}$ : 322.1199; found: 322.1196; ${ }^{1} \mathrm{H}$ NMR $\left(\mathrm{CDCl}_{3}, 300\right.$ $\mathrm{MHz}): \delta=3.92(\mathrm{~s}, 6 \mathrm{H}), 6.72-6.80(\mathrm{~m}, 2 \mathrm{H}), 7.01-7.11(\mathrm{~m}, 2 \mathrm{H}), 7.50\left(\mathrm{~d},{ }^{3} J=8.5 \mathrm{~Hz}, 4 \mathrm{H}\right)$, $8.00\left(\mathrm{~d},{ }^{3} J=8.5 \mathrm{~Hz}, 4 \mathrm{H}\right) ;{ }^{13} \mathrm{C} \mathrm{NMR}\left(\mathrm{CDCl}_{3}, 100 \mathrm{MHz}\right): \delta=52.0\left(\mathrm{CH}_{3}\right), 126.3(\mathrm{CH})$, $129.1(\mathrm{C}), 130.0(\mathrm{CH}), 131.2(\mathrm{CH}), 133.1(\mathrm{CH}), 141.4(\mathrm{C}), 166.7(\mathrm{C})$. 
$(1 E, 3 E, 5 E, 7 E)-1,8-d i p h e n y l o c t a-1,3,5,7-t e t r a e n e(4 \mathrm{~g}):$

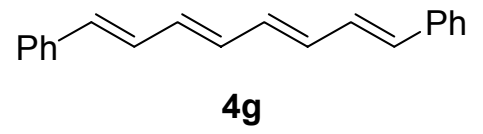

HRMS (EI): calcd. for $\mathrm{C}_{20} \mathrm{H}_{18}$ : 258.1403; found: 258.1407; ${ }^{1} \mathrm{H} \mathrm{NMR}\left(\mathrm{CDCl}_{3}, 400\right.$ MHz): $\delta=6.47-6.48(\mathrm{~m}, 4 \mathrm{H}), 6.60\left(\mathrm{~d},{ }^{3} J_{\text {trans }}=15.5 \mathrm{~Hz}, 2 \mathrm{H}\right), 6.86-6.91(\mathrm{~m}, 2 \mathrm{H}) ; 7.23(\mathrm{t}$, $\left.{ }^{3} J=7.2 \mathrm{~Hz}, 2 \mathrm{H}\right), 7.33\left(\mathrm{t},{ }^{3} J=7.2 \mathrm{~Hz}, 4 \mathrm{H}\right), 7.43\left(\mathrm{~d},{ }^{3} J=7.2 \mathrm{~Hz}, 4 \mathrm{H}\right) ;{ }^{13} \mathrm{C} \mathrm{NMR}\left(\mathrm{CDCl}_{3}\right.$, $100 \mathrm{MHz}): \delta=126.3(\mathrm{CH}), 127.5(\mathrm{CH}), 128.6(\mathrm{CH}), 129.1(\mathrm{CH}), 132.6(\mathrm{CH}), 133.4$ (CH), $137.3(\mathrm{C})$.

${ }^{1} \mathrm{H}$ and ${ }^{13} \mathrm{C}$ NMR spectra of all the compounds 3 and 4 prepared: 

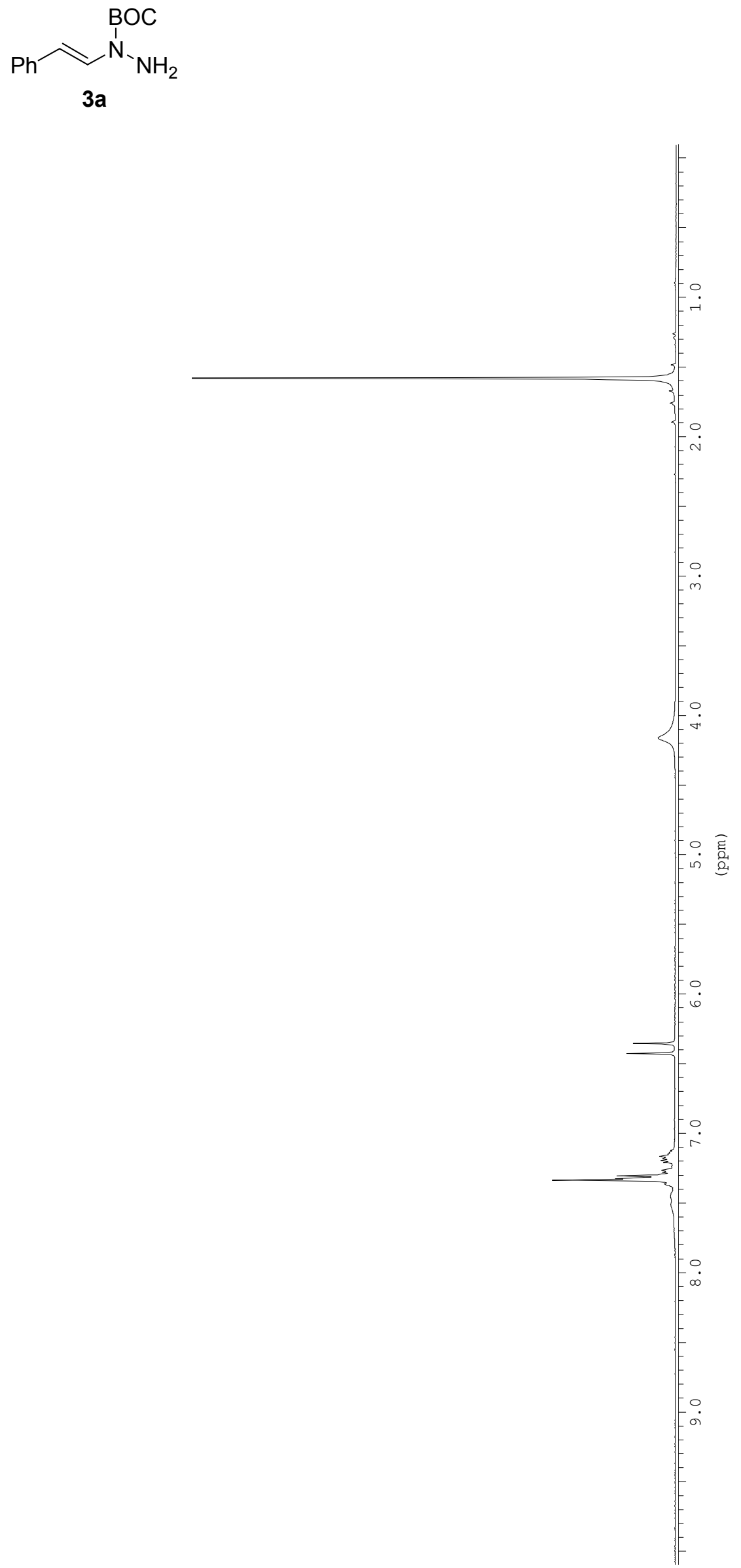


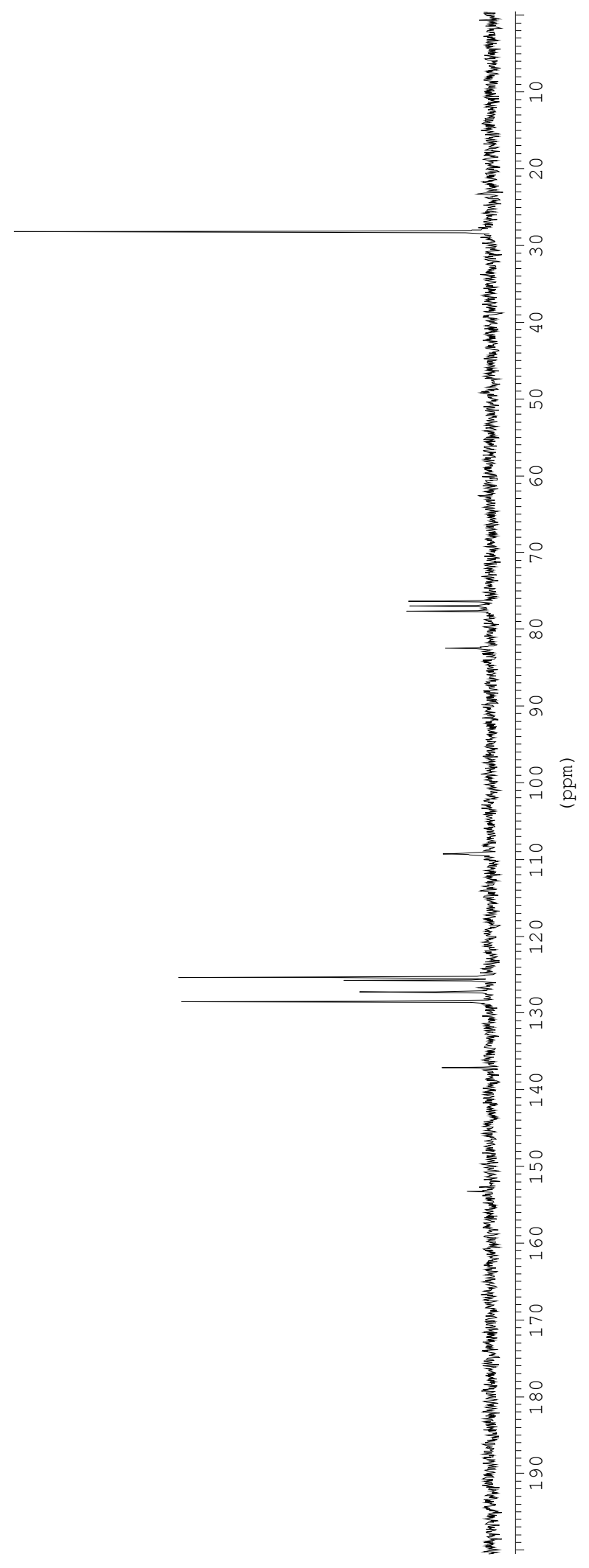




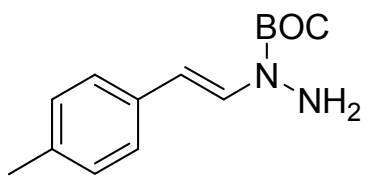

$3 b$

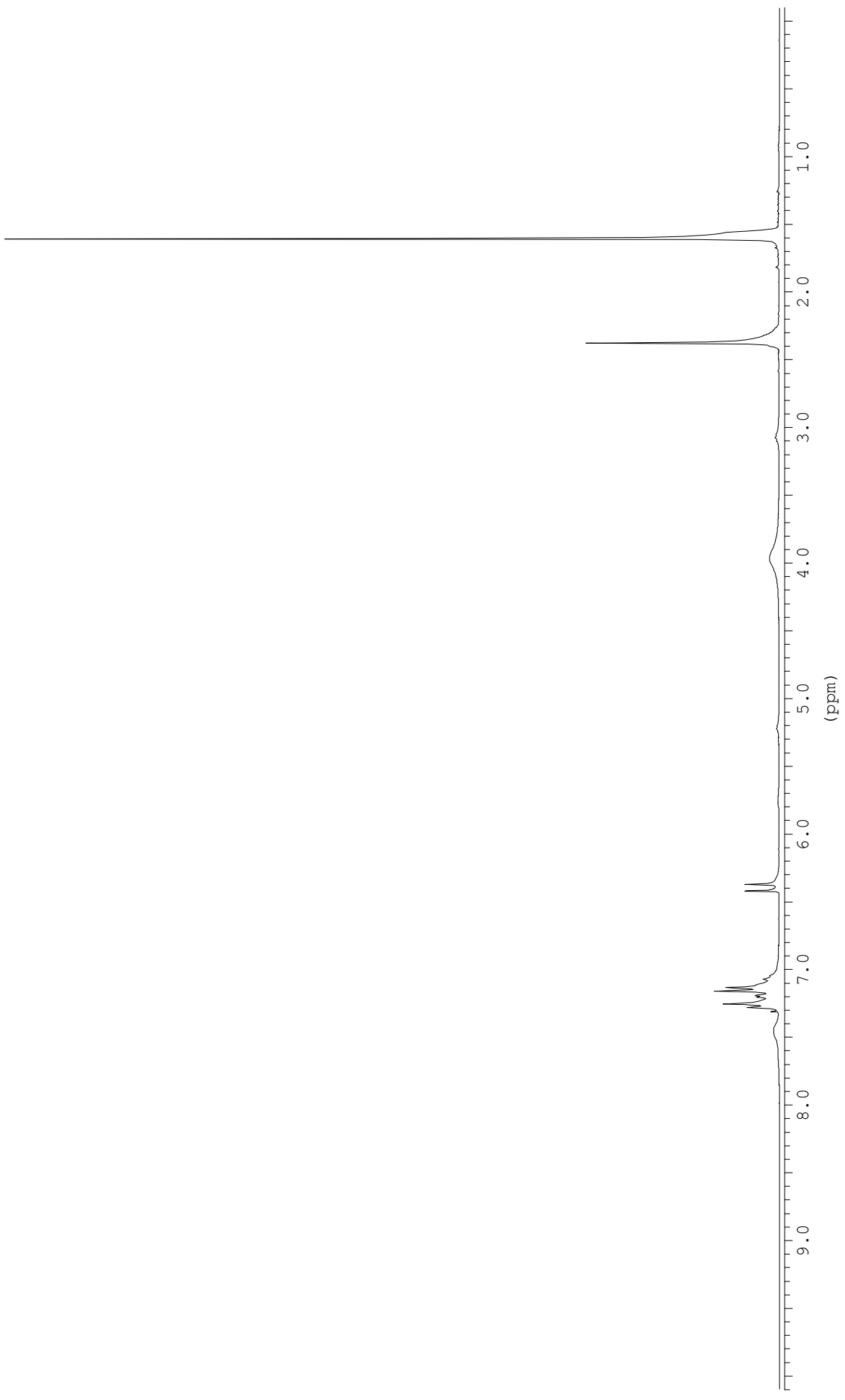




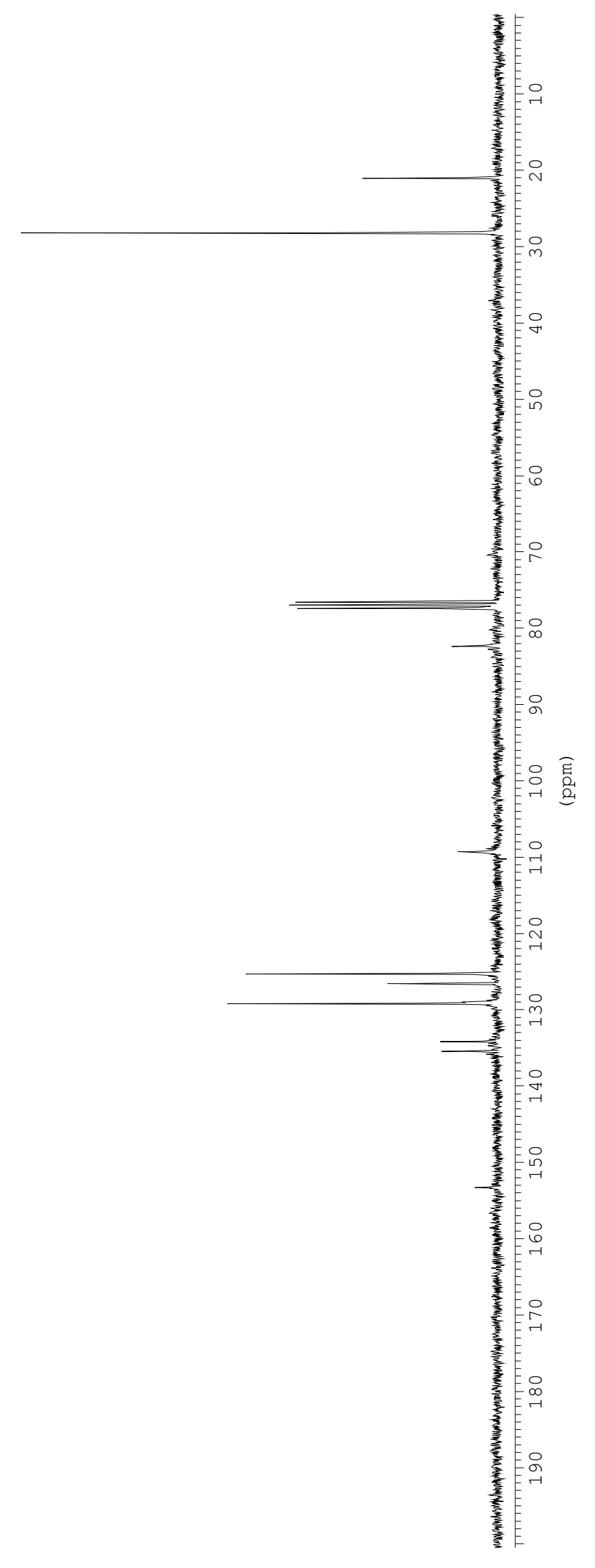



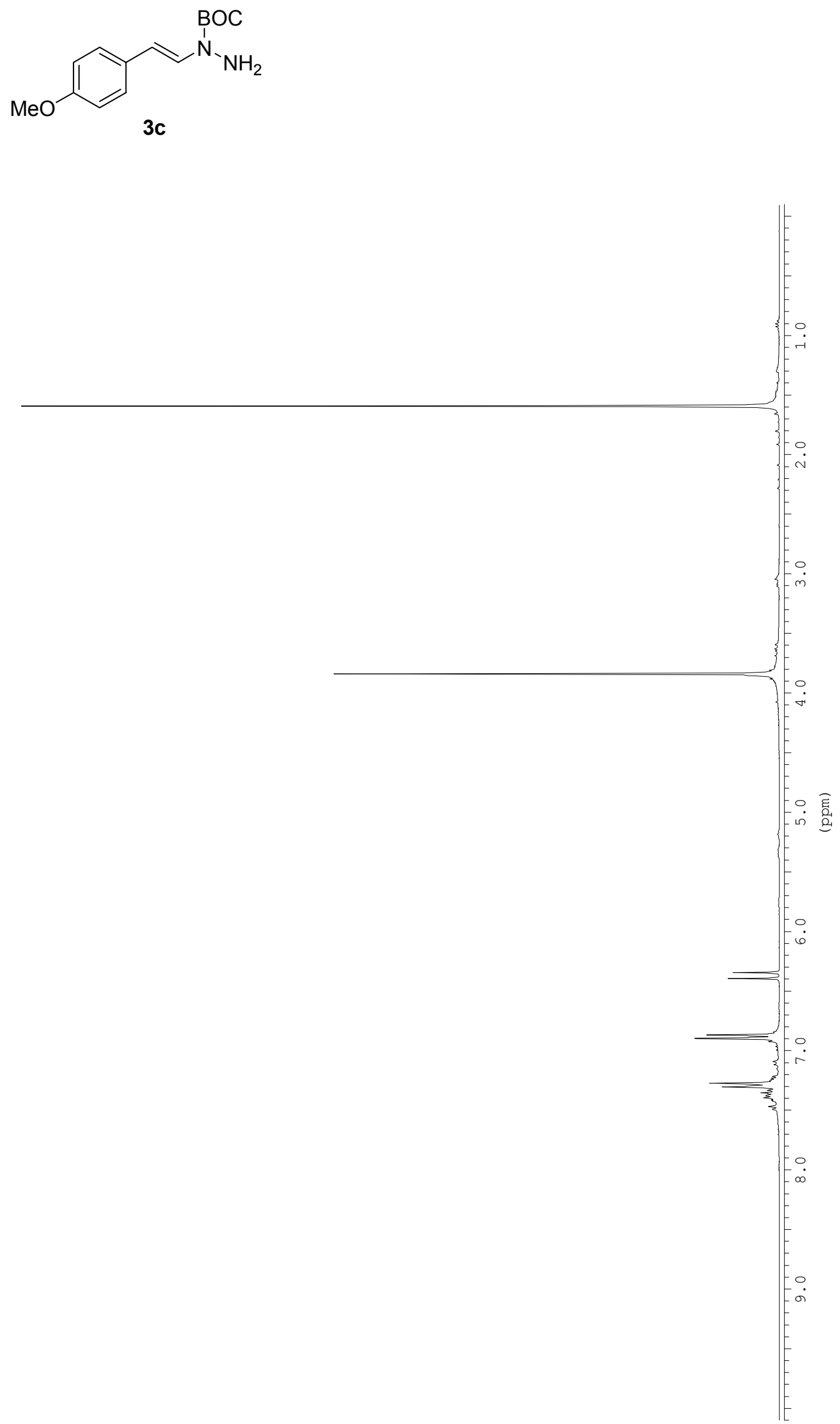


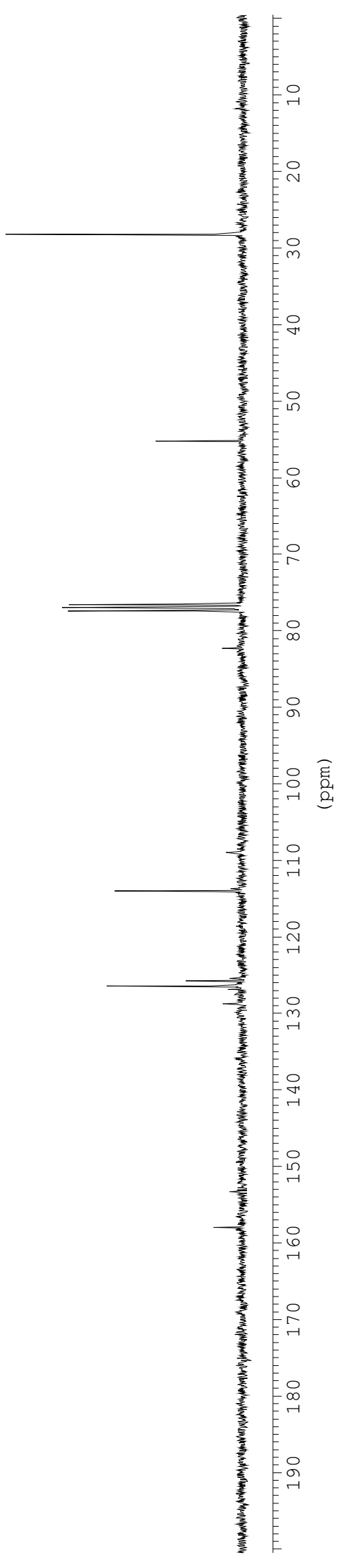

S14 

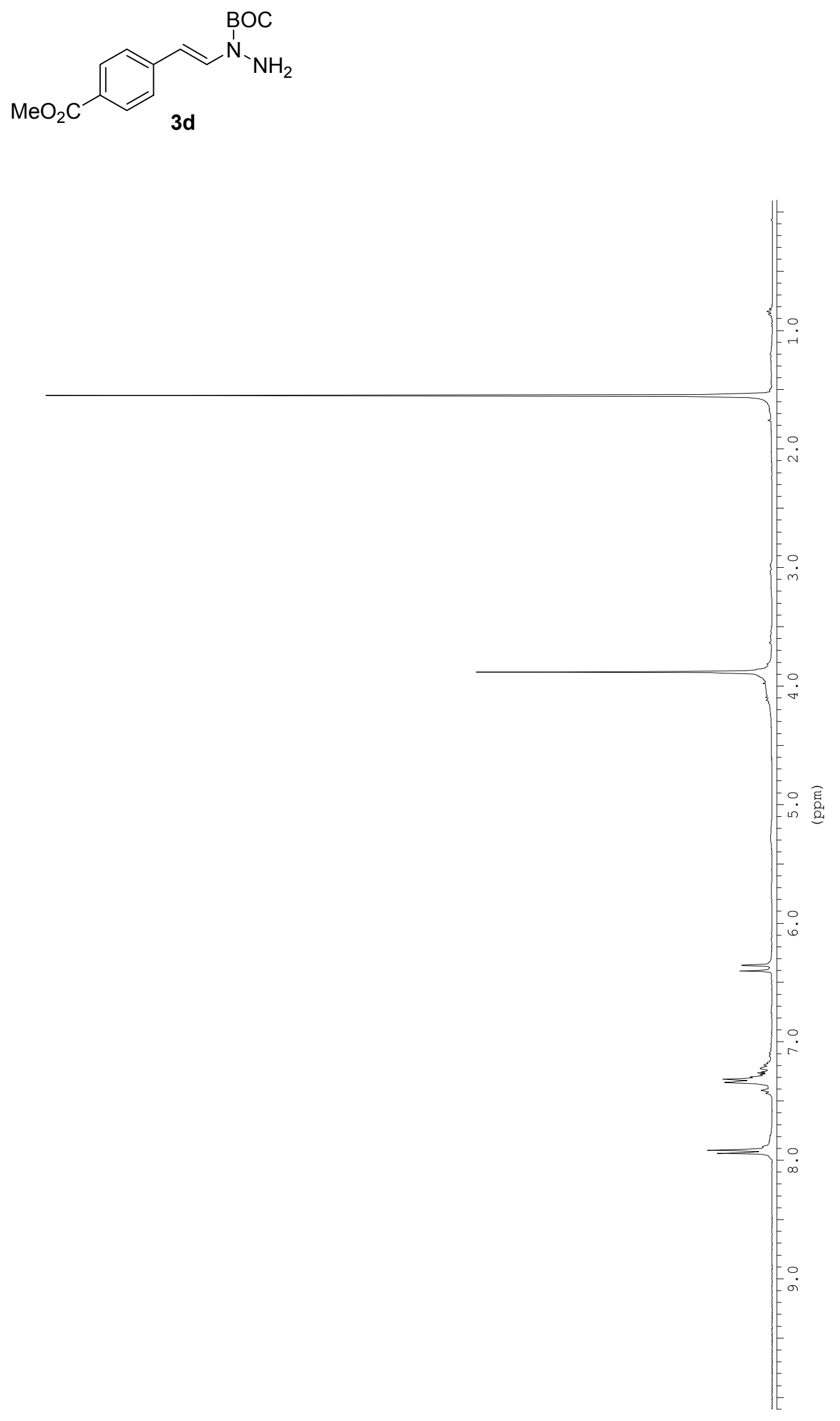


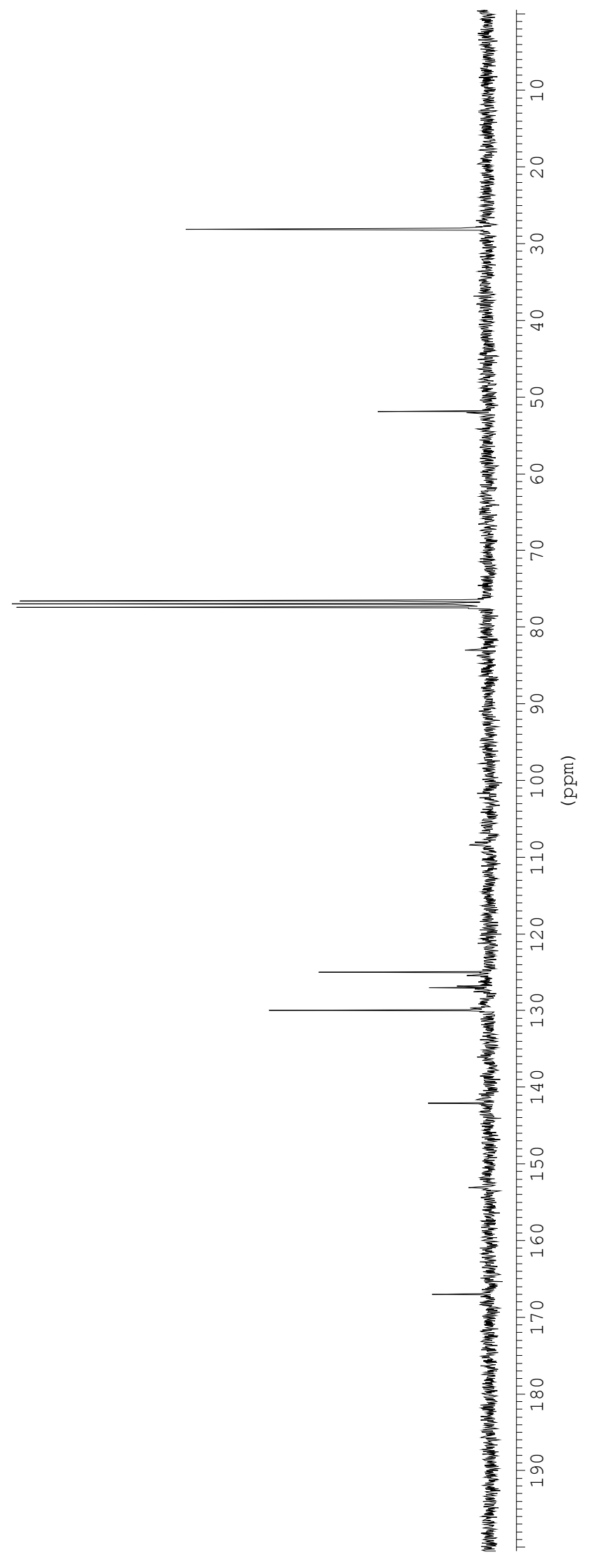

S16 

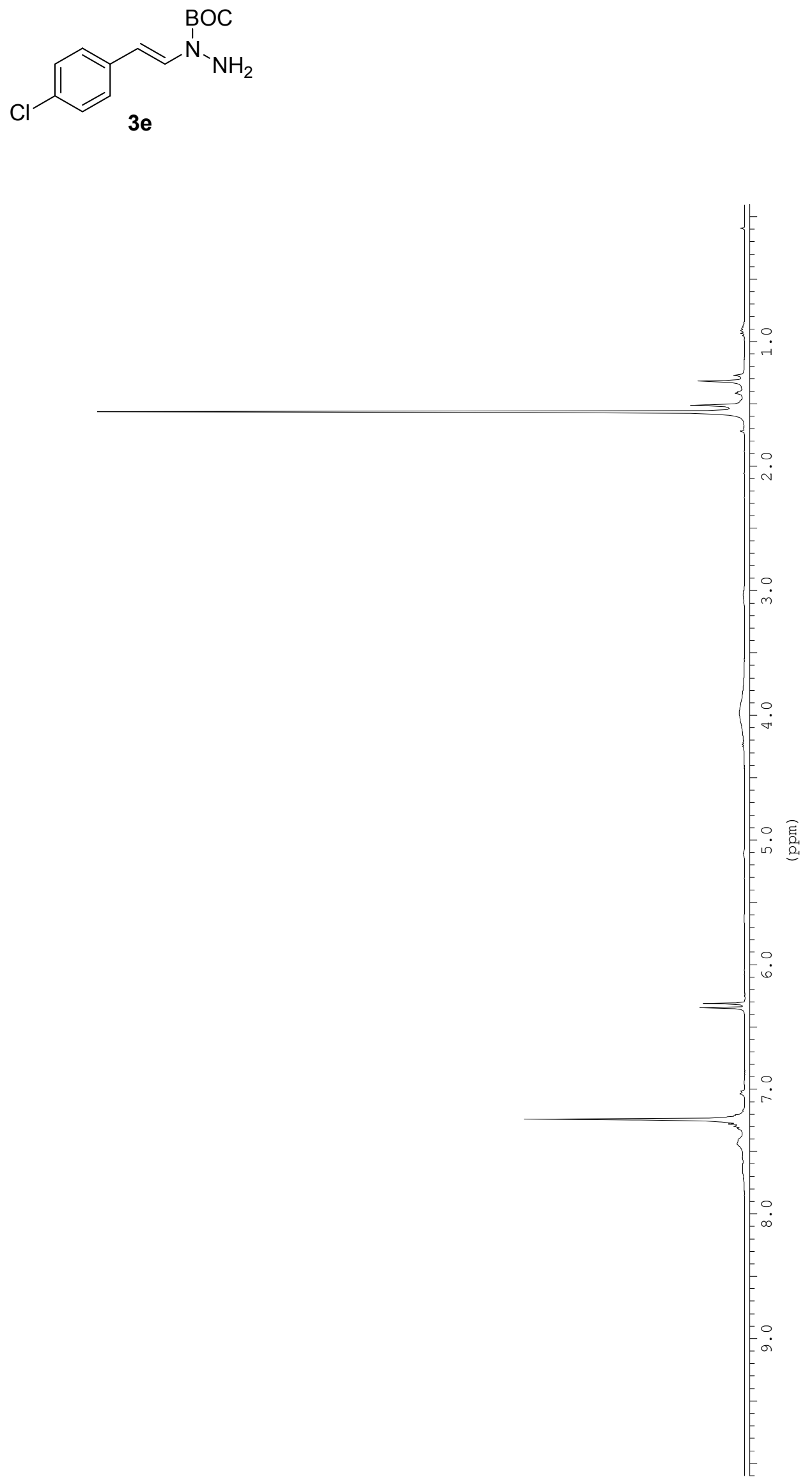


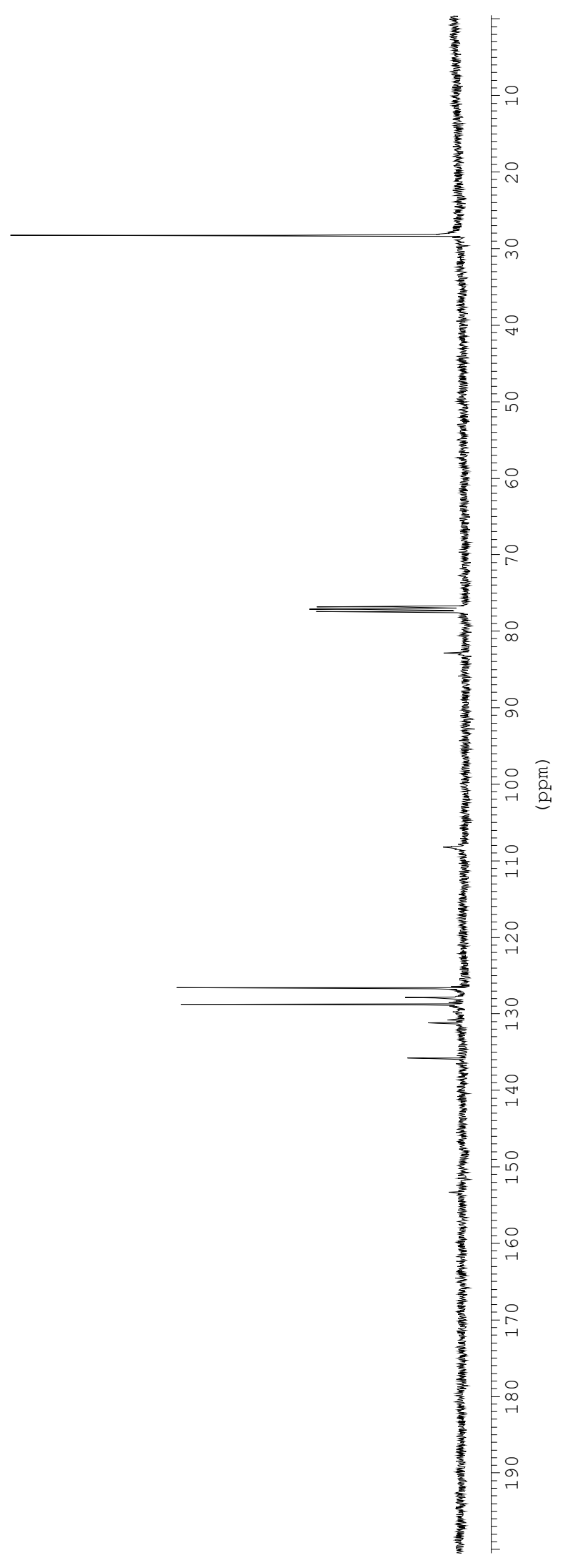



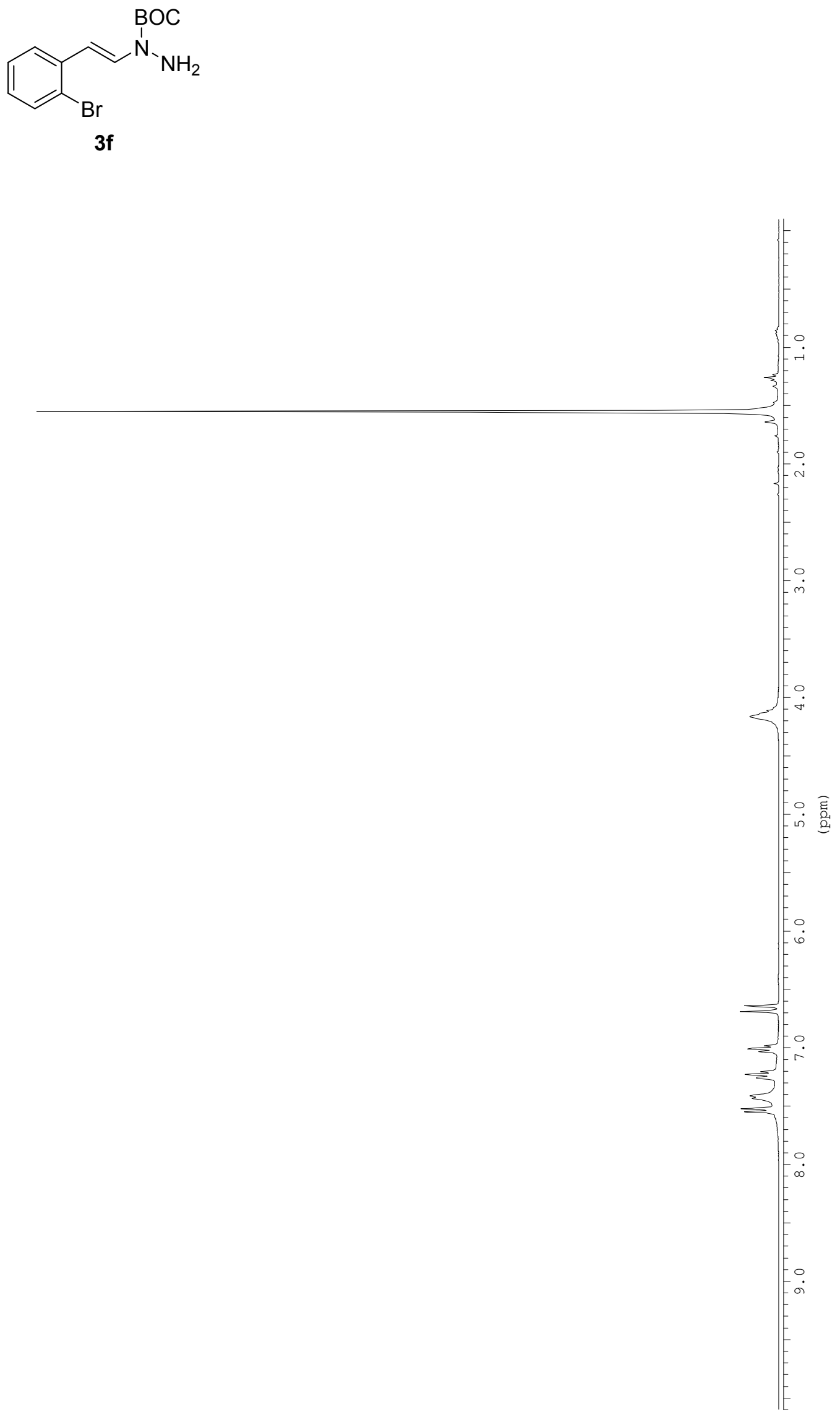


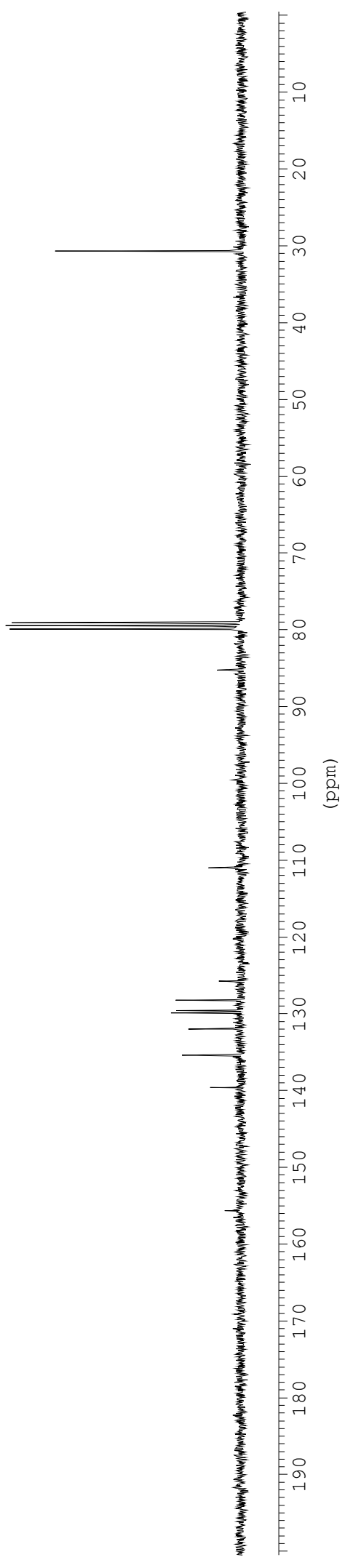




$$
\overbrace{\mathrm{Nh}_{-}^{\mathrm{BOC}}}^{\mathrm{BOC}}
$$

$3 g$

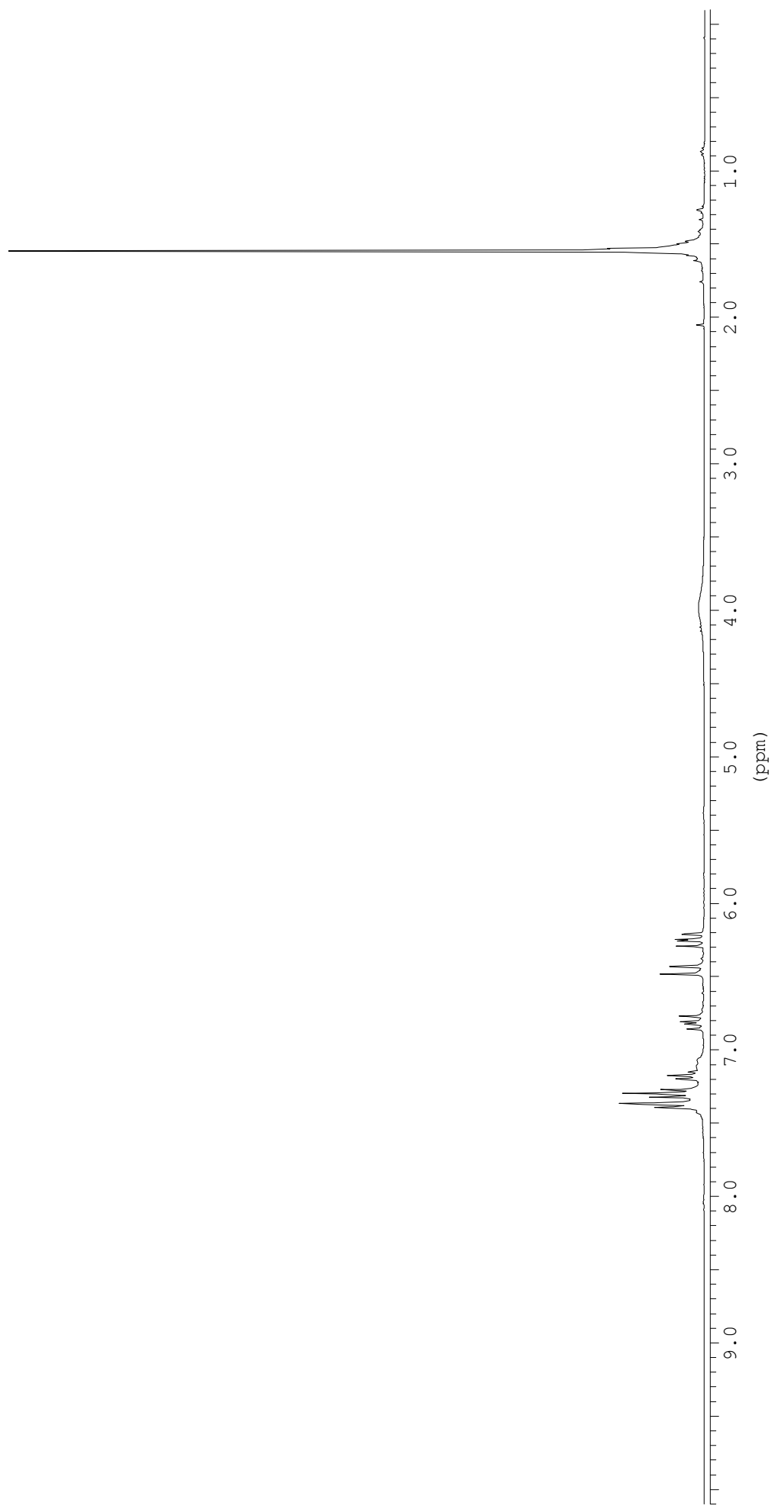




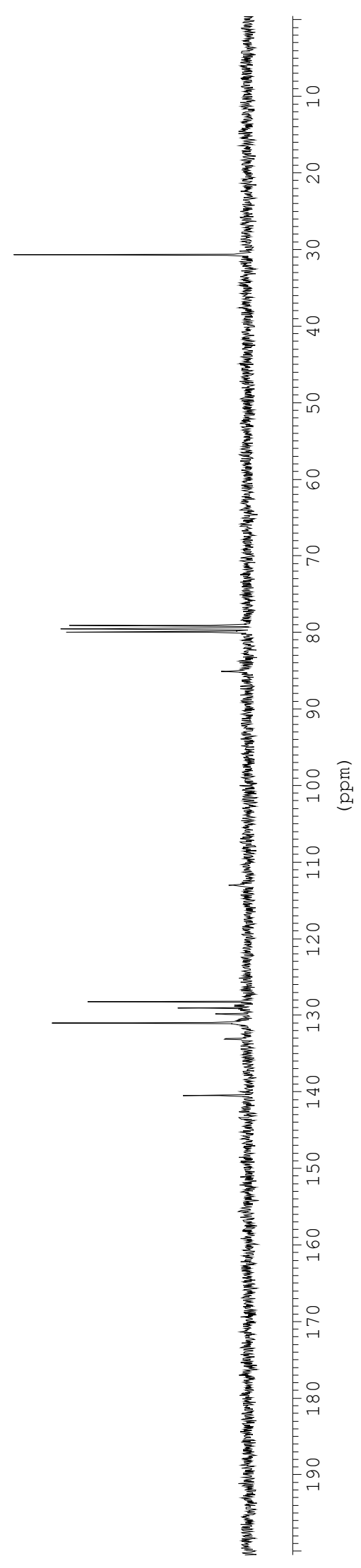



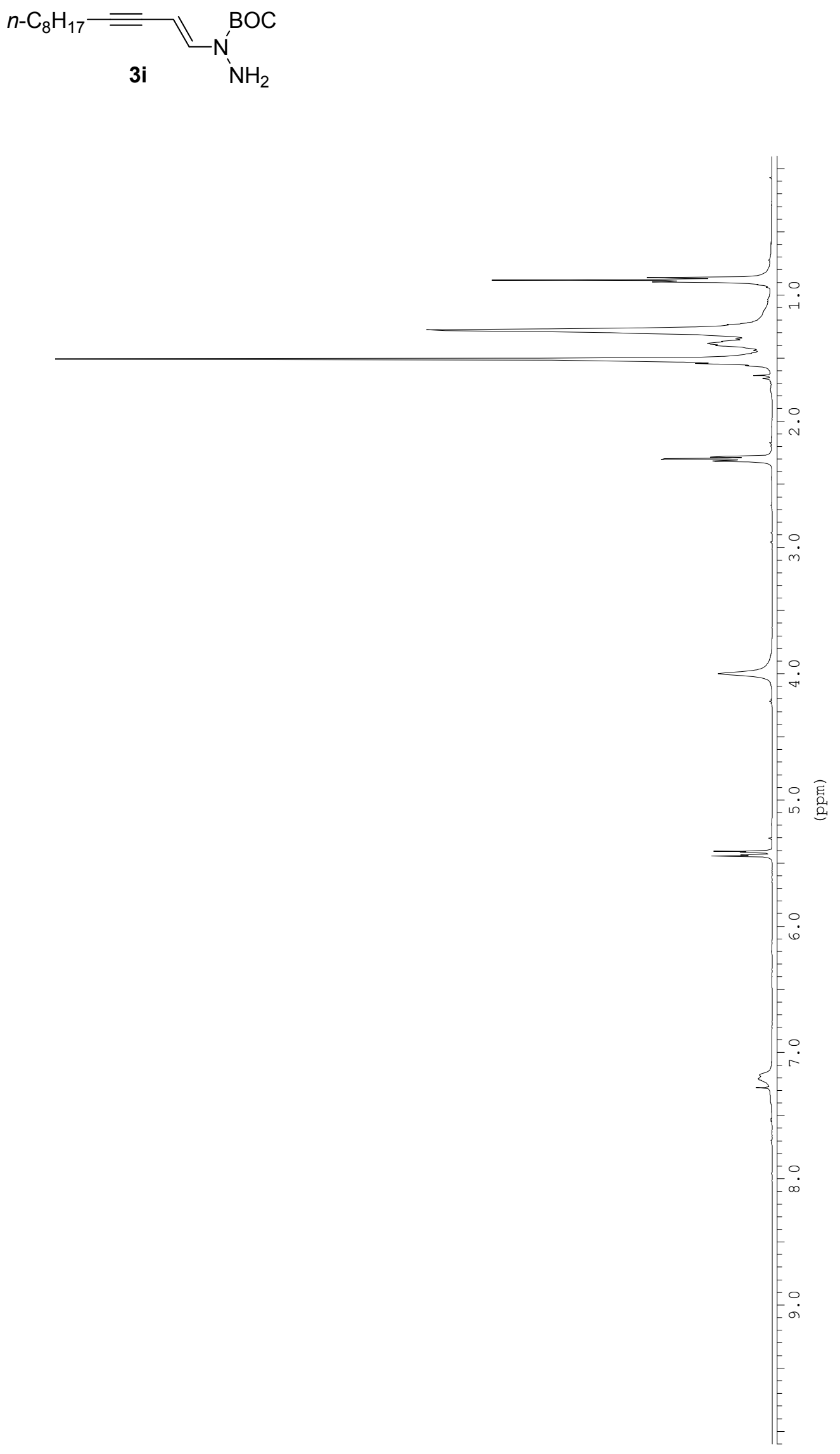


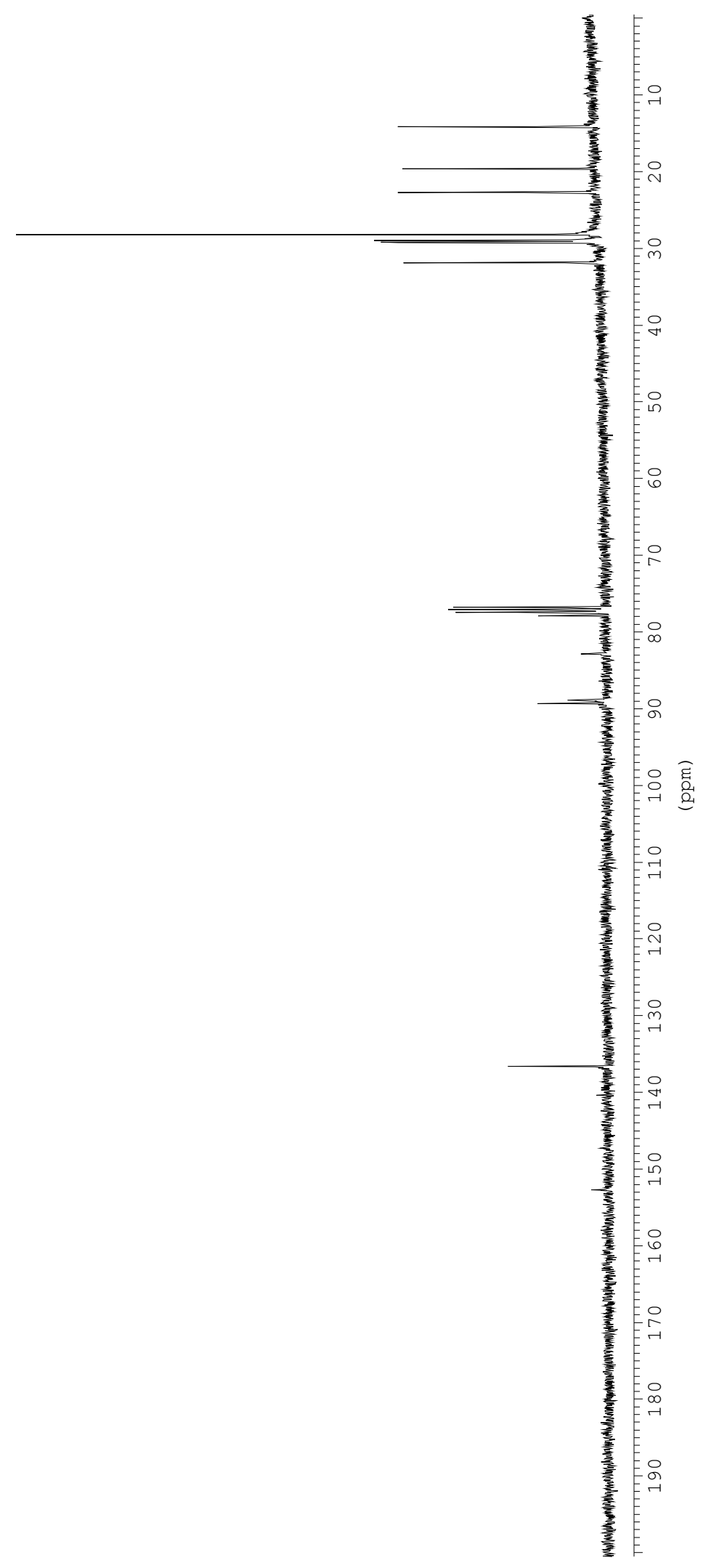




$$
n-\mathrm{C}_{4} \mathrm{H}_{9} \curvearrowright \stackrel{\substack{1 \\ \mathrm{~N}}}{{ }_{N H_{2}}}
$$

3i

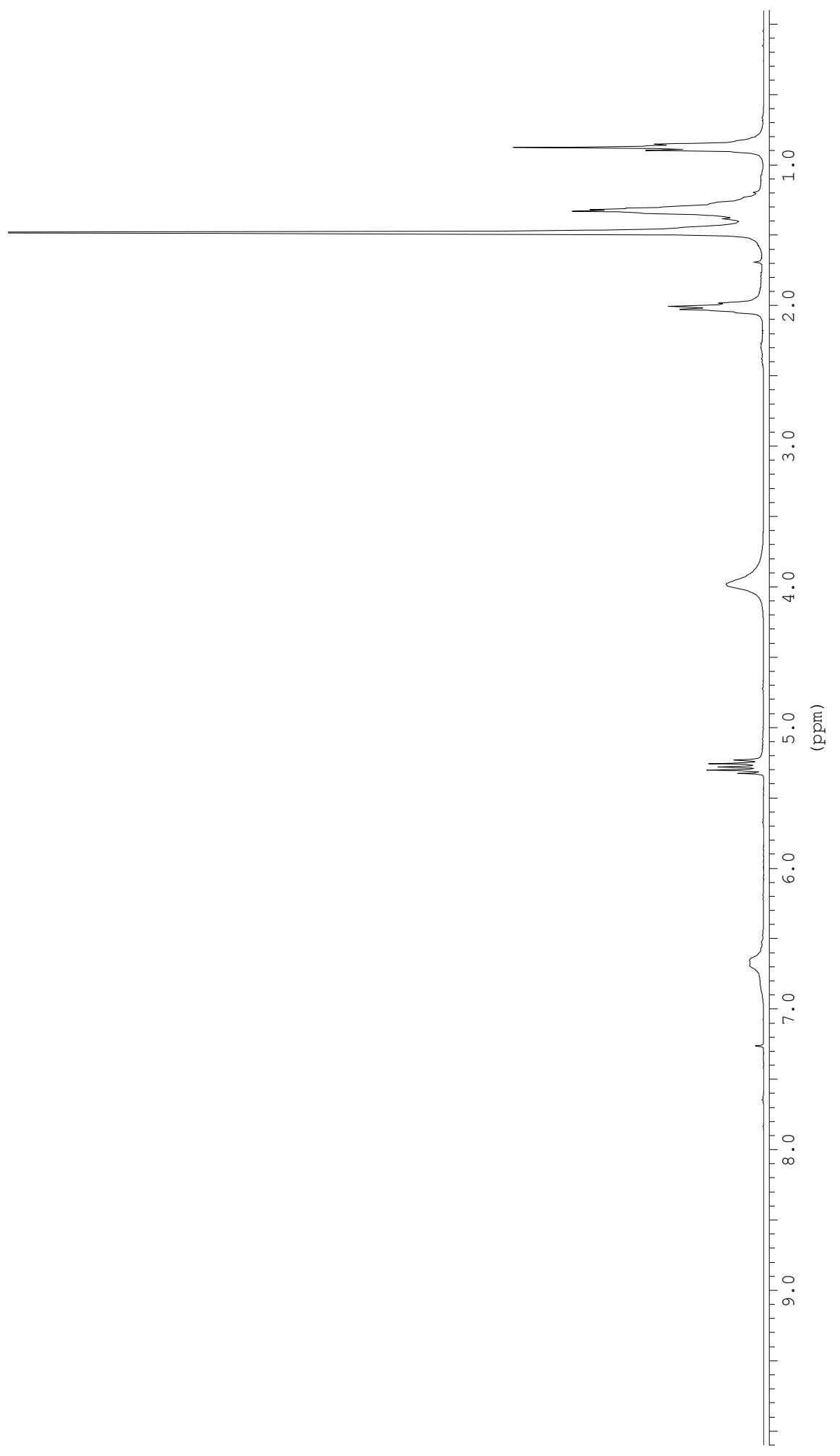




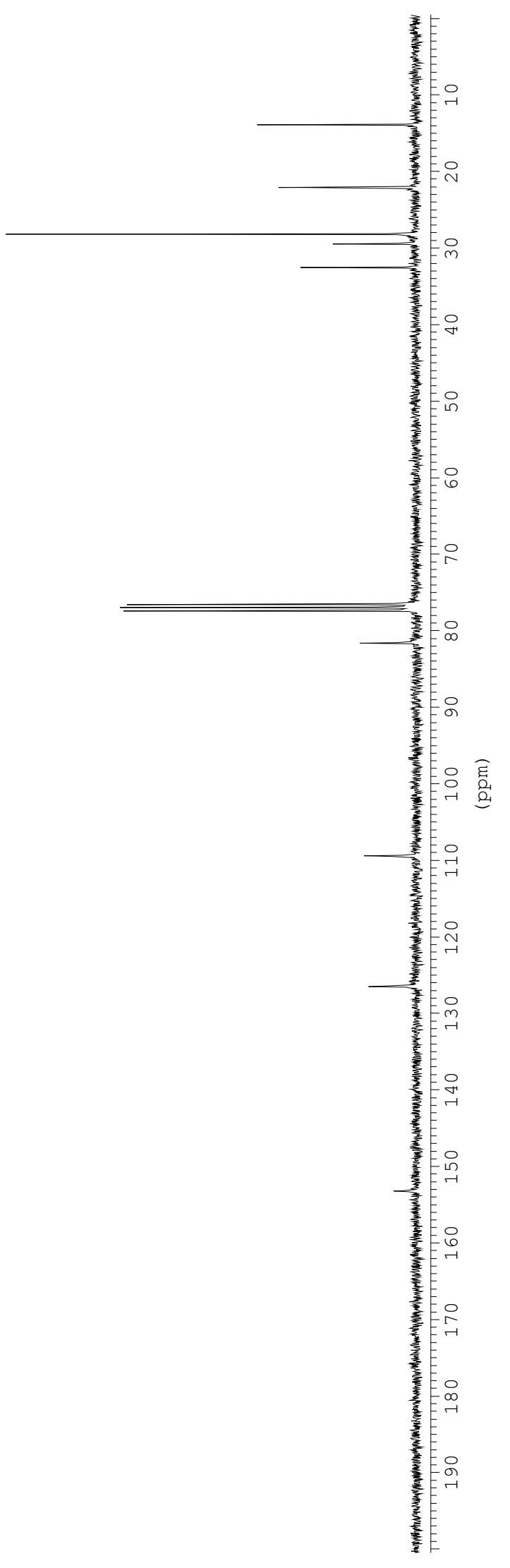




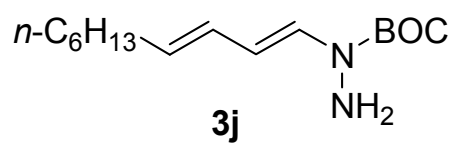

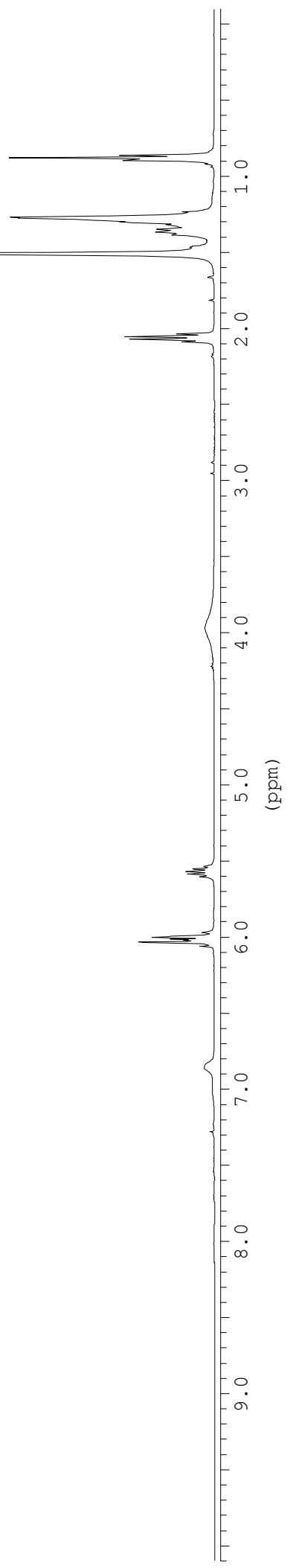




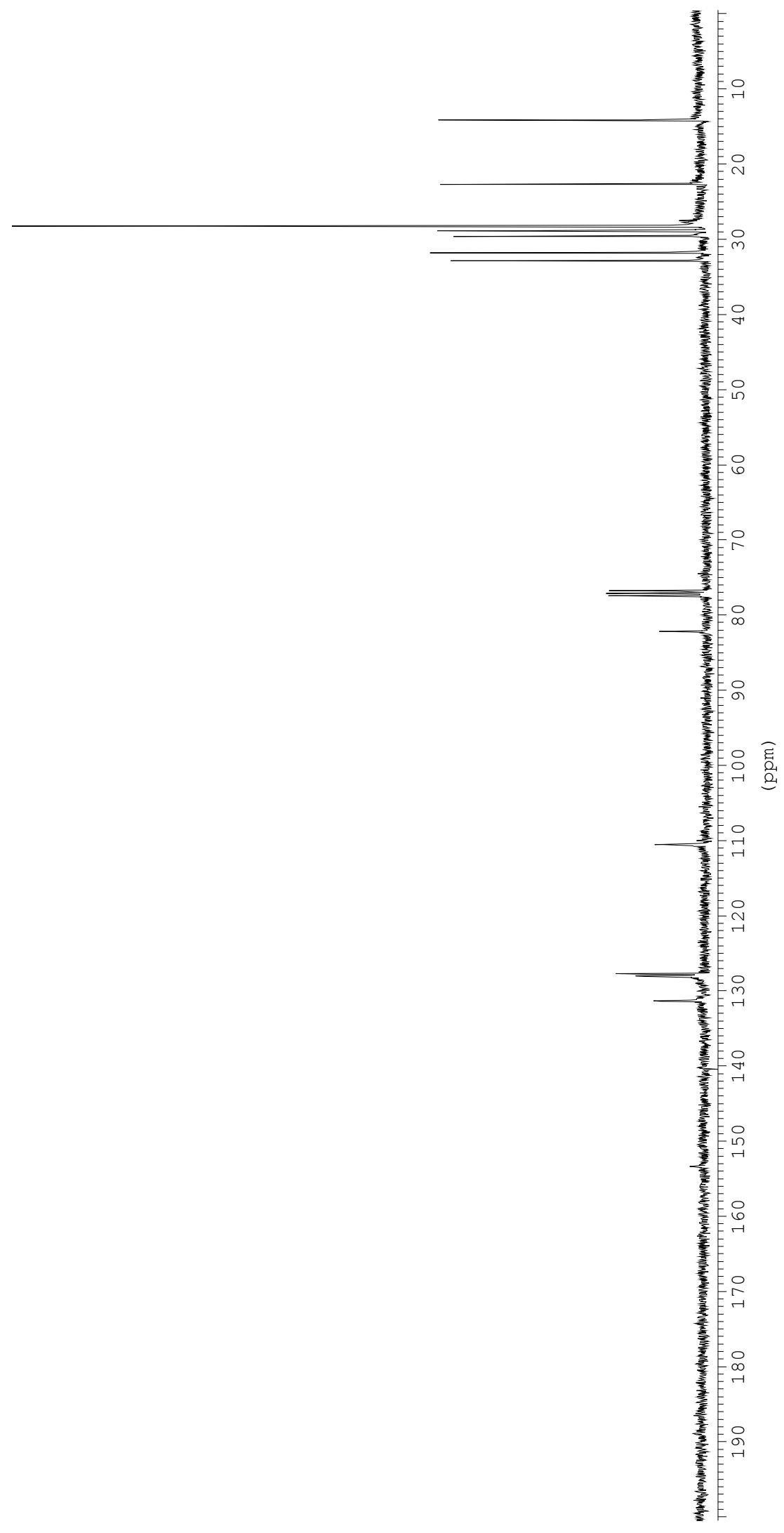



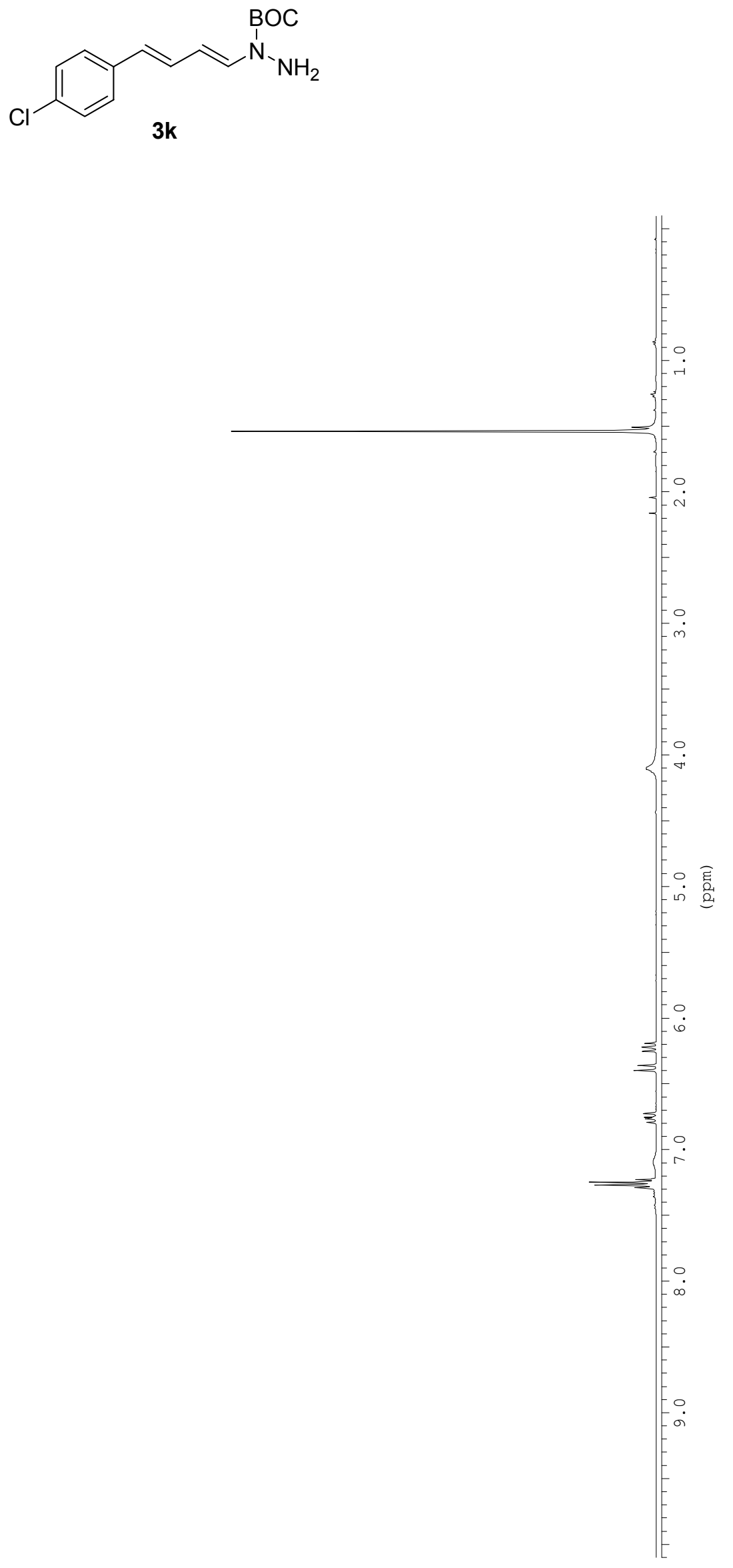


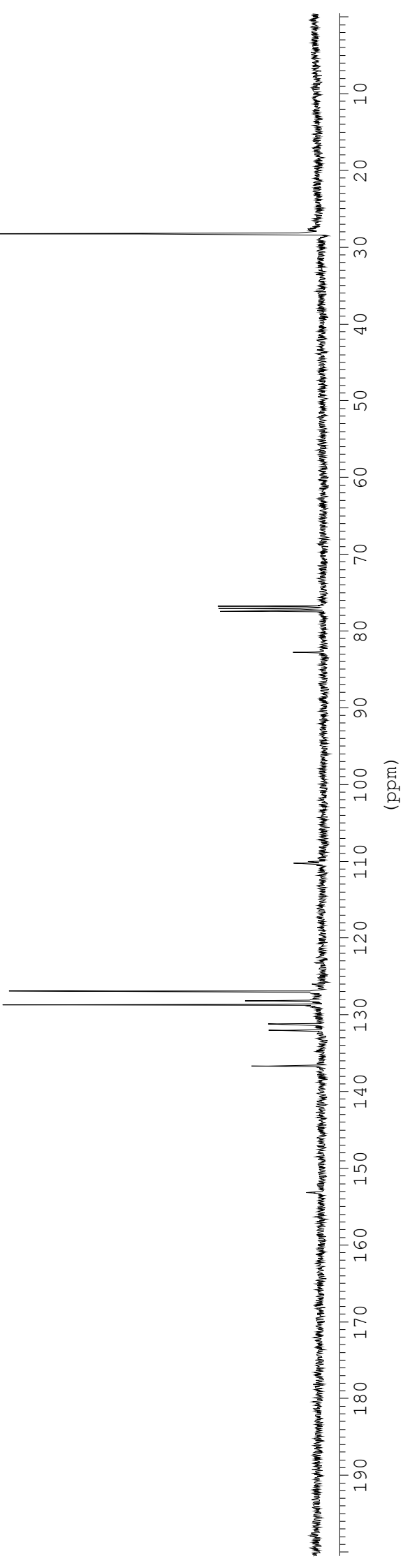


$\mathrm{Ph} /{ }_{\mathrm{Ph}}$

$4 a$

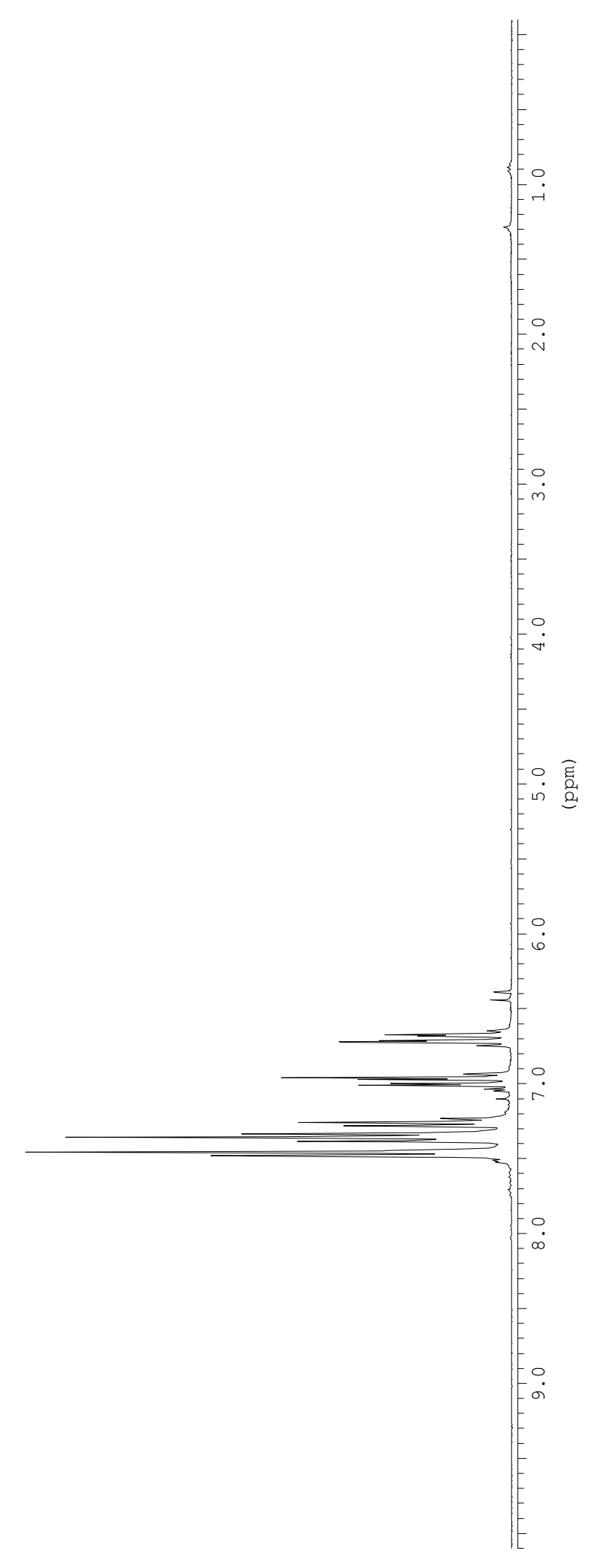




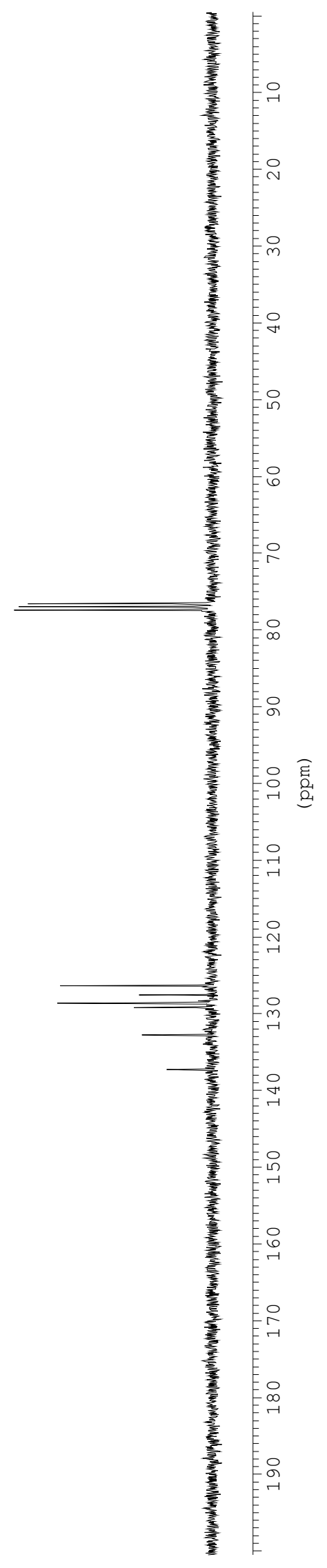

S32 


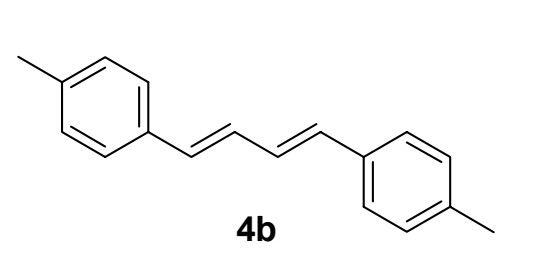




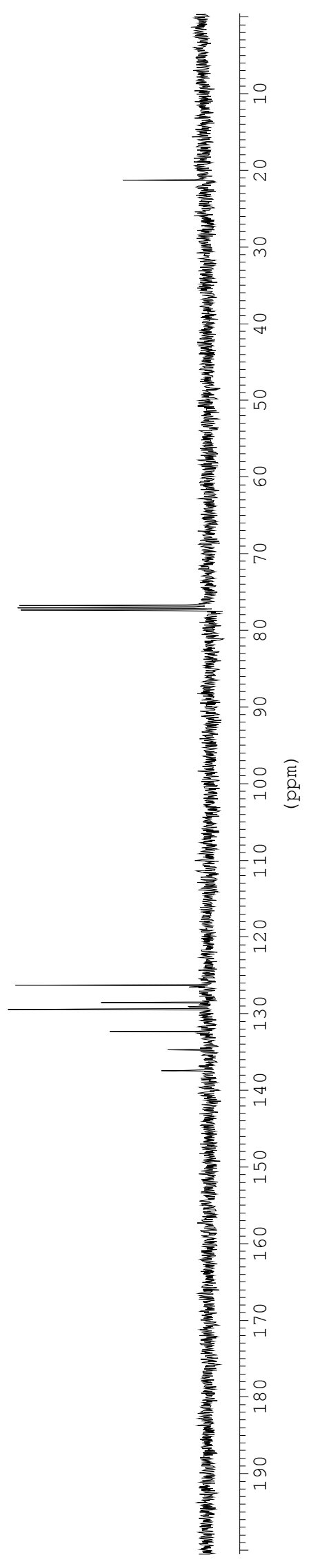

S34 
(dd

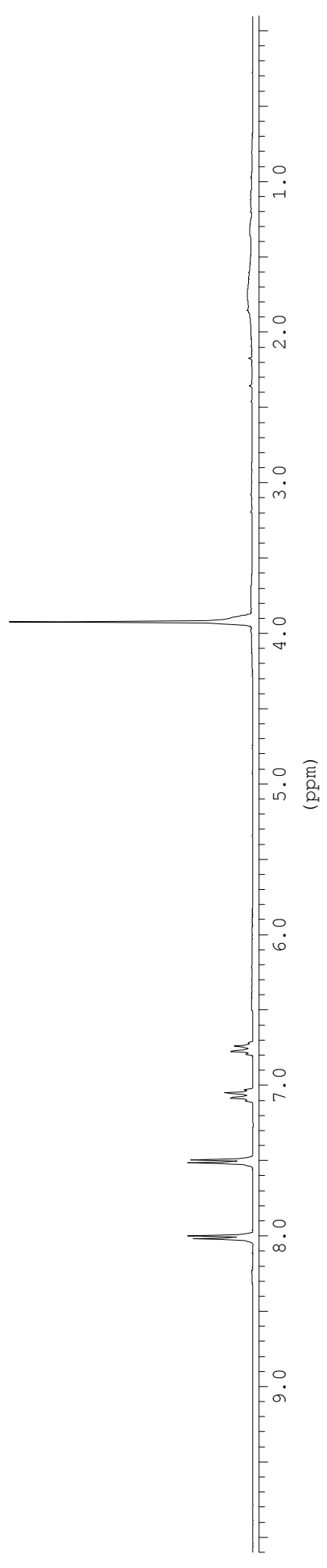




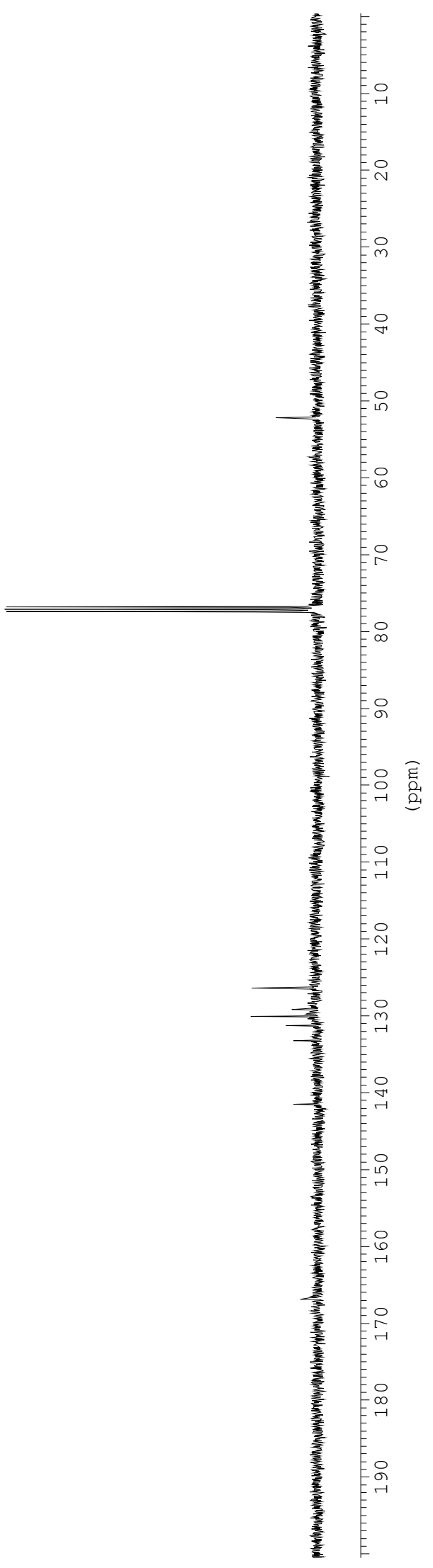




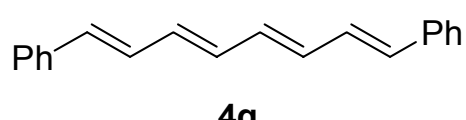

$4 \mathrm{~g}$

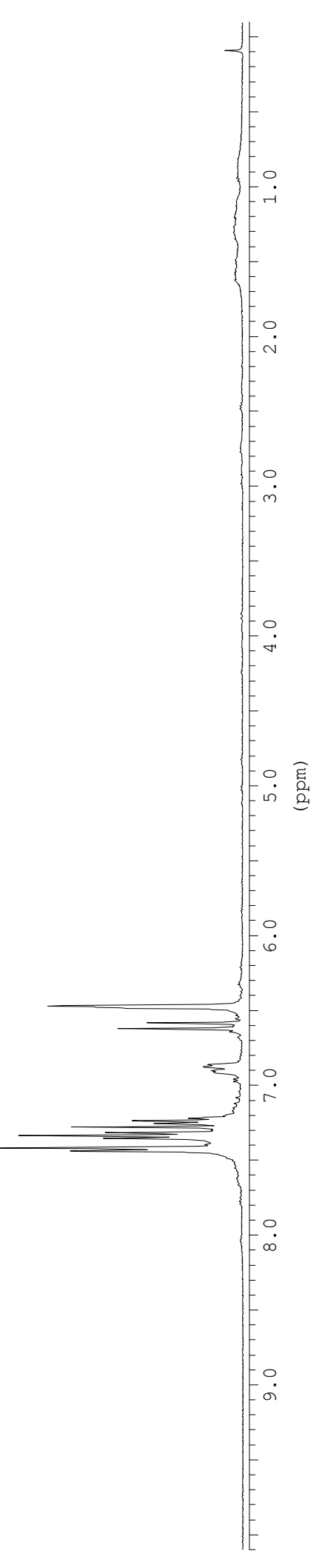




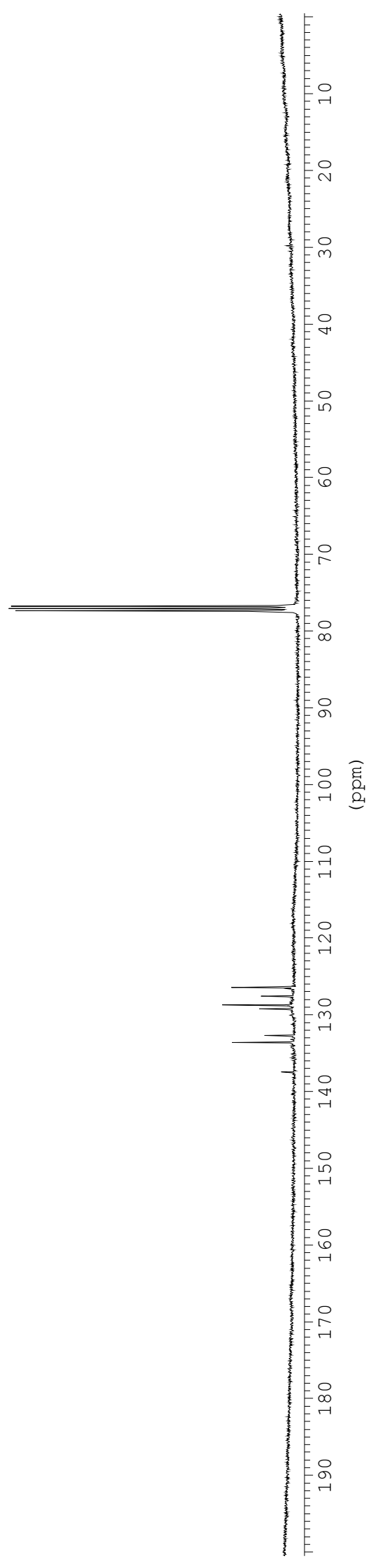

